\title{
Flavor hierarchies from dynamical scales
}

\author{
Giuliano Panico ${ }^{a}$ and Alex Pomarol ${ }^{a, b, c}$ \\ ${ }^{a}$ IFAE and BIST, Universitat Autònoma de Barcelona, \\ Bellaterra, Barcelona, 08193 Spain \\ ${ }^{b}$ CERN, Theory Division, \\ Geneva 23, CH-1211 Switzerland \\ ${ }^{c}$ Dept. de Física, Universitat Autònoma de Barcelona, \\ Bellaterra, Barcelona, 08193 Spain \\ E-mail: gpanico@ifae.es, alex.pomarol@uab.cat
}

ABSTRACT: One main obstacle for any beyond the SM (BSM) scenario solving the hierarchy problem is its potentially large contributions to electric dipole moments. An elegant way to avoid this problem is to have the light SM fermions couple to the BSM sector only through bilinears, $\bar{f} f$. This possibility can be neatly implemented in composite Higgs models. We study the implications of dynamically generating the fermion Yukawa couplings at different scales, relating larger scales to lighter SM fermions. We show that all flavor and $\mathrm{CP}$-violating constraints can be easily accommodated for a BSM scale of few $\mathrm{TeV}$, without requiring any extra symmetry. Contributions to $B$ physics are mainly mediated by the top, giving a predictive pattern of deviations in $\Delta F=2$ and $\Delta F=1$ flavor observables that could be seen in future experiments.

Keywords: Beyond Standard Model, Effective field theories, Higgs Physics, Technicolor and Composite Models

ARXIV EPRINT: 1603.06609 


\section{Contents}

1 Introduction 1

2 Multiple flavor scales in composite Higgs models 4

3 Implications in flavor and CP-violation physics $\quad 8$

$3.1 \Delta F=2$ transitions 8

$\begin{array}{lll}3.2 & \text { Neutron EDM } & 12\end{array}$

$\begin{array}{ll}3.3 \Delta F=1 \text { transitions } & 12\end{array}$

3.4 Electron EDM, $\mu \rightarrow e \gamma$ and $\tau \rightarrow \mu \gamma \quad 13$

4 Alternative scenarios $\quad 15$

5 Higgs couplings to fermions $\quad 17$

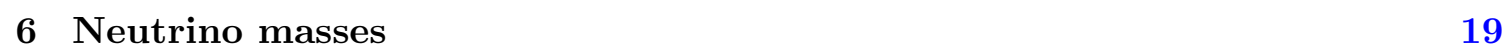

$\begin{array}{llr}7 & \text { Conclusions } & 20\end{array}$

A Warped five-dimensional models with multiple flavor scales 22

B Mixing angles in the $\alpha_{L}^{d s, d b, s b} \approx 0$ limit $\quad 22$

\section{Introduction}

An attractive solution to the hierarchy problem is to require that the Higgs is not an elementary particle, but a composite state arising from some strongly-coupled sector at $\mathrm{TeV}$ energies. This possibility has important implications for the theory of flavor. Contrary to models with an elementary Higgs in which the structure of Yukawa couplings can have its origin at very high energies, as large as the Planck scale, in composite Higgs models the origin of flavor must be addressed at much lower energies. This is because the Higgs is associated with a composite operator of the strong sector $\mathcal{O}_{H}$ whose dimension $d_{H}$ must be larger than one to avoid the hierarchy problem, ${ }^{1}$ implying that $\bar{f}_{L} \mathcal{O}_{H} f_{R}$ has dimension larger than 4, that is to say that the Yukawa couplings are irrelevant at low energies. Therefore, if $\bar{f}_{L} \mathcal{O}_{H} f_{R}$ are generated at very high energies, e.g. the Planck scale, fermion masses will be too small at the electroweak scale.

\footnotetext{
${ }^{1}$ For the hierarchy problem what is in fact needed is that the dimension of the gauge-singlet term $\mathcal{O}_{H} \mathcal{O}_{H}^{\dagger}$ is larger than $\sim 4$, to avoid relevant operators in the theory. In strongly-coupled theories with a large- $N$ expansion this implies $d_{H} \geq 2$, but this is not true in general. Nevertheless, bounds from conformal bootstrap [1] indicate that it is not possible to have $d_{H} \sim 1$ together with $\operatorname{Dim}\left[\mathcal{O}_{H} \mathcal{O}_{H}^{\dagger}\right] \gtrsim 4$. Being conservative, we will be considering here $d_{H} \gtrsim 2$.
} 
Different approaches to flavor in composite Higgs models have been considered. The most popular one is partial compositeness, in which the SM fermions $f_{i}$ get masses by mixing linearly with an operator of the strong sector:

$$
\mathcal{L}_{\text {lin }}=\epsilon_{f_{i}} \bar{f}_{i} \mathcal{O}_{f_{i}} .
$$

At the strong scale $\Lambda_{\mathrm{IR}} \sim \mathrm{TeV}$, which determines the mass-gap of the model, and at which the Higgs emerges as a composite state, the fermion Yukawa couplings are generated with a pattern

$$
\mathcal{Y}_{f} \sim g_{*} \epsilon_{f_{i}} \epsilon_{f_{j}}
$$

where $1<g_{*} \lesssim 4 \pi$ characterizes the coupling in the strong sector. The appealing feature of these scenarios, usually called "anarchic partial compositeness" [2-6], is the fact that the smallness of the mixing $\epsilon_{f_{i}}$ can simultaneously explain the smallness of the fermion masses and mixing angles. Nevertheless, this approach also predicts flavor-violating higherdimensional operators of order [7]

$$
\frac{g_{*}^{2}}{16 \pi^{2}} \frac{g_{*} v}{\Lambda_{\mathrm{IR}}^{2}} \epsilon_{f_{i}} \epsilon_{f_{j}} \bar{f}_{i} \sigma_{\mu \nu} f_{j} g F^{\mu \nu}, \quad \frac{g_{*}^{2}}{\Lambda_{\mathrm{IR}}^{2}} \epsilon_{f_{i}} \epsilon_{f_{j}} \epsilon_{f_{k}} \epsilon_{f_{l}} \bar{f}_{i} \gamma^{\mu} f_{j} \bar{f}_{k} \gamma_{\mu} f_{l},
$$

where $v \simeq 174 \mathrm{GeV}$. The operators in eq. (1.3) lead for $\Lambda_{\mathrm{IR}} \sim \mathrm{TeV}$ to large contributions to the electron and neutron electric dipole moment (EDM), $\mu \rightarrow e \gamma$ and $\epsilon_{K}$, above the experimental bounds [8] (see also refs. [9-17]), as shown in table 3. Taking $\Lambda_{\mathrm{IR}}$ above the $\mathrm{TeV}$ is possible, but at the price of fine-tuning the electroweak scale. ${ }^{2}$

An interesting alternative to the above approach is to consider the right-handed quarks to be fully composite $[20,21]$. If the strong sector has an accidental SU(3) flavor symmetry and $\mathrm{CP}$ symmetry (something not difficult to envisage as it occurs in QCD), the flavor bounds can be easily satisfied. Indeed, in this case the whole flavor structure comes only from the linear mixing of the left-handed fermions with the strong sector that must then be proportional to the SM Yukawas $\mathcal{Y}_{f}$, as in models with minimal flavor violation (MFV) [22]. Therefore flavor bounds are easily satisfied for $\Lambda_{\mathrm{IR}} \sim \mathrm{TeV}$. Nevertheless, due to the compositeness of the right-handed quarks, 4-fermion contact interactions, as for example,

$$
\frac{g_{*}^{2}}{\Lambda_{\mathrm{IR}}^{2}}\left(\bar{u}_{R} \gamma_{\mu} u_{R}\right)^{2}
$$

lead to large deviation in dijets distributions, $p p \rightarrow j j$, at high energies, and sizable production cross sections for composite resonances in the multi-TeV mass range are predicted [2325]. All these effects have not been observed at the LHC and severely constrain these models. Similar results can be found in variations of these ideas with other composite SM fermions [26-32].

Wrapping up, composite Higgs models must address the SM flavor structure at low energies, giving then unequivocal predictions for flavor observables. The models proposed

\footnotetext{
${ }^{2}$ Alternative constructions have been recently proposed based on composite Twin Higgs in which the scale of compositeness can be pushed up without introducing additional tuning in the Higgs potential [18]. It is also possible to reduce some bounds by taking smaller $g_{*}$, but this implies reducing the UV cutoff (see for example ref. [19]).
} 
so far seem to clash with some experimental data. Although extra flavor and CP symmetries could be imposed, for example in the mixing terms $\epsilon_{f_{i}}$, to avoid certain experimental bounds, it is unclear how these symmetries could emerge in the model. One needs to specify the dynamics of the model to understand whether flavor and CP symmetries can arise accidentally at low energies.

Here we would like to put forward a deviation from the anarchic paradigm that can avoid these severe flavor and CP-violating constraints. The idea is to assume that the operators $\mathcal{O}_{f_{i}}$ of eq. (1.1), that mediate the mixing between the SM fermions and the Higgs, get an effective mass at some energy scale $\Lambda_{f_{i}} \gg \Lambda_{\mathrm{IR}} \sim \mathrm{TeV}$, and then decouple from the strong sector. This implies that Yukawa-like couplings

$$
\mathcal{L}_{\text {bil }} \sim \bar{f}_{i} \mathcal{O}_{H} f_{j}
$$

are generated at scales larger than $\Lambda_{\mathrm{IR}}$, avoiding in this way sizable contributions to flavor and CP-violating observables. The hierarchies in the fermion spectrum of the SM and the small flavor mixing angles could be now explained by the different scales $\Lambda_{f_{i}}$ instead of the small $\epsilon_{f_{i}}$. The larger the $\Lambda_{f_{i}}$, the smaller the Yukawa coupling for $f_{i}$. Without imposing any extra symmetry in the model, we will derive by simple power-counting which are the strongest flavor and CP-violating constraints, independently of the details of the models. We find that top-mediated processes give the largest contribution to flavor-violating observables. These are characterized by only two operators. One operator generates the $\Delta F=2$ processes $\epsilon_{K}, \Delta M_{B_{d}}$ and $\Delta M_{B_{s}}$ at a level close to the present experimental constraints for $\Lambda_{\mathrm{IR}} \sim$ few $\mathrm{TeV}$. The second operator leads to flavor-violating $Z$-couplings, contributing simultaneously to $K \rightarrow \mu^{+} \mu^{-}, \epsilon^{\prime} / \epsilon, B \rightarrow(X) \ell \ell$ and $Z \rightarrow b \bar{b}$ with a size also close to the experimental bounds. There are also important contributions arising from the scale at which the charm and strange masses are generated, $10^{7}-10^{8} \mathrm{GeV}$, leading also to sizable effects to $\epsilon_{K}$, and forcing $d_{H} \lesssim 2$. Contributions to the neutron EDM are dominated by the top EDM, being not far from the present experimental bound. On the other hand, in the lepton sector we find that the dominant contribution to the electron EDM comes at the two-loop level from Barr-Zee type diagrams [33], and is around the experimental bound, while $\mu \rightarrow e \gamma$ is found to be very small. Therefore these scenarios provide realistic examples where the flavor and hierarchy problem can be dynamically solved without contradicting the present experimental data, and which near future experiments could be able to explore. Having proposed a different origin for fermion masses, we also analyze the expected deviations in Higgs couplings.

Our approach to the small fermion masses is a reminiscent of the old ExtendedTechnicolor idea [34, 35], in which masses from eq. (1.5) were generated from an extended gauge sector, or from integrating heavy fermions [36]. Earlier attempts along these lines were considered recently in refs. [37, 38] for composite Higgs models. In these models, however, Yukawa-like couplings were generated at a single energy scale, and the light quark families were connected by potentially large mixing angles. This leads to additional sizable new-physics effects and to bounds typically more stringent than the ones we find here. Furthermore, the lepton sector, where the experimental bounds are the most difficult to satisfy, was not considered. 
The attempt of this work is to show that flavor bounds can be satisfied in composite Higgs models without the need of imposing flavor symmetries. We do not provide an explanation for the hierarchies in the fermion masses, as these are just traded for the scales $\Lambda_{f_{i}}$. Nevertheless, it is not hard to imagine a possible mechanism that explains the largeness of $\Lambda_{f_{i}}$, thus providing a reason for the smallness of the light-generations masses. For example, it is possible to envisage scenarios where the $\Lambda_{f_{i}}$ could be generated from dimensional transmutation, explaining in this way the size of the fermion masses as a function of $O(1)$ couplings. We will not pursue further the origin of $\Lambda_{f_{i}}$, but assume that they have the correct values to fit the SM fermion masses.

We would like to close this section by stressing that in most scenarios beyond the SM (BSM) that address the hierarchy problem, including supersymmetry, one generically finds large EDMs. This is because fermions have linear couplings to BSM fields. For example, in supersymmetric models fermions couple linearly to sfermions and gauginos, leading generically to sizable EDMs at the one-loop level. Therefore, unless ad hoc symmetries are imposed in the BSM sector, the only way to avoid these large contributions is to restrict the SM fermions to have bilinear couplings to the BSM states, as the scenarios proposed here. In this case the dominant contributions to EDMs arise at the two-loop level (see diagram figure 3) that can be accommodated just below the experimental constraint.

\section{Multiple flavor scales in composite Higgs models}

Our framework for flavor shares many features of previous composite Higgs models with partly-composite fermions via eq. (1.1). The main crucial difference is the assumption that the operators $\mathcal{O}_{f_{i}}$, which are the portals of the SM fermions to the strong sector, decouple at some scale $\Lambda_{f_{i}}$, generating the Yukawa terms $\bar{f}_{L} \mathcal{O}_{H} f_{R}$ at that scale instead of at $\Lambda_{\mathrm{IR}}$ as in the anarchic case. The decoupling of the operator $\mathcal{O}_{f_{i}}$ can be due to the fact that some of the constituents of $\mathcal{O}_{f_{i}}$ get a mass $\sim \Lambda_{f_{i}}$, or that a dynamically generated mass-gap makes heavy all composite states created by $\mathcal{O}_{f_{i}}$ (those $|\Psi\rangle$ with $\left\langle 0\left|\mathcal{O}_{f_{i}}\right| \Psi\right\rangle \neq 0$ ). Using the AdS/CFT correspondence, we can easily visualize this type of scenarios by warped extradimensional models with several branes, as the example shown in figure 5 of appendix A. In what follows we will estimate the flavor structure of these scenarios without restricting to any specific UV realization.

The scale at which the Yukawa coupling for the SM fermion $f=u, d, e, \ldots$ is generated is determined by the scale $\Lambda_{f}$ at which either $\mathcal{O}_{f_{R}}$ or $\mathcal{O}_{f_{L}}$ decouple from the strong sector. We choose these scales following figure 1 . This is our dynamical assumption. No further symmetries will be imposed. Other options could also be possible, and we will consider later more economical models with fewer scales $\Lambda_{f}$. Under the assumption of figure 1 , the Yukawa structure will be the following. Let us consider first the down-type quark sector. At the lowest scale $\Lambda_{b}$, we have only one pair of operators $\mathcal{O}_{Q_{L 3}}$ and $\mathcal{O}_{b_{R}}$, to which only one linear combination of SM left-handed and right-handed quarks can respectively mix with. We name these linear combinations the 3rd family left-handed quark, $Q_{L 3}$, and right-handed bottom, $b_{R}$ :

$$
\mathcal{L}_{\text {lin }}^{(3)}=\epsilon_{b_{L}}^{(3)} \bar{Q}_{L 3} \mathcal{O}_{Q_{L 3}}+\epsilon_{b_{R}}^{(3)} \bar{b}_{R} \mathcal{O}_{b_{R}}
$$




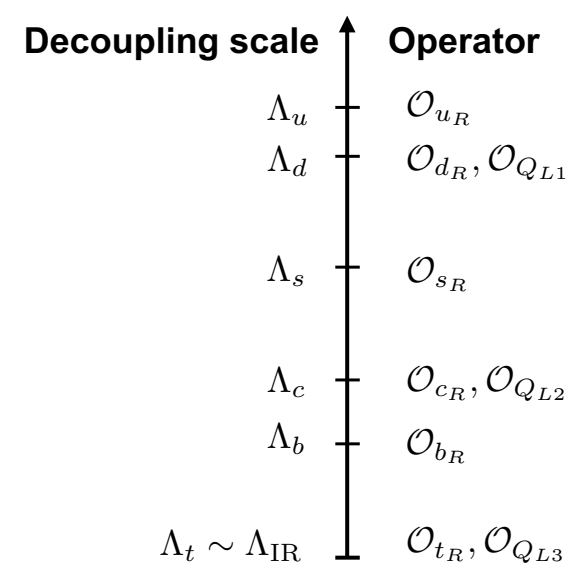

Figure 1. Energy scale at which the fermionic operators $\mathcal{O}_{f_{i}}$ decouple from the strong sector.

Below $\Lambda_{b}$, after integrating out $\mathcal{O}_{b_{R}}$, the following Yukawa-like operator is expected to be generated

$$
\mathcal{L}_{\text {bil }}^{(3)}=\frac{1}{\Lambda_{b}^{d_{H}-1}}\left(\epsilon_{b_{L}}^{(3)} \bar{Q}_{L 3}\right) \mathcal{O}_{H}\left(\epsilon_{b_{R}}^{(3)} b_{R}\right),
$$

where $\mathcal{O}_{H}$ corresponds to the lowest-dimensional operator that at $\Lambda_{\mathrm{IR}}$ projects into the Higgs, $\left\langle 0\left|\mathcal{O}_{H}\right| H\right\rangle \neq 0$, and $d_{H}$ is its energy dimension. At a larger scale $\Lambda_{s} \gg \Lambda_{b}$, we have another pair of operators $\mathcal{O}_{Q_{L 2}}$ and $\mathcal{O}_{s_{R}}$ present, coupled to a different linear combination of $\mathrm{SM}$ fermions. By an $\mathrm{SU}(3)$ rotation that does not affect eq. (2.2) we can always go to the basis where this linear combination contains only two quarks, $Q_{L 3}$ and $Q_{L 2}$ (this latter is identified with the second family left-handed quark), and similarly for the right-handed sector, $b_{R}$ and $s_{R}$ :

$$
\mathcal{L}_{\text {lin }}^{(2)}=\left(\epsilon_{b_{L}}^{(2)} \bar{Q}_{L 3}+\epsilon_{s_{L}}^{(2)} \bar{Q}_{L 2}\right) \mathcal{O}_{Q_{L 2}}+\left(\epsilon_{b_{R}}^{(2)} b_{R}+\epsilon_{s_{R}}^{(2)} s_{R}\right) \mathcal{O}_{s_{R}}
$$

that below $\Lambda_{s}$, after integrating $\mathcal{O}_{s_{R}}$, leads to

$$
\mathcal{L}_{\mathrm{bil}}^{(2)}=\frac{1}{\Lambda_{s}^{d_{H}-1}}\left(\epsilon_{b_{L}}^{(2)} \bar{Q}_{L 3}+\epsilon_{s_{L}}^{(2)} \bar{Q}_{L 2}\right) \mathcal{O}_{H}\left(\epsilon_{b_{R}}^{(2)} b_{R}+\epsilon_{s_{R}}^{(2)} s_{R}\right)
$$

Finally, at $\Lambda_{d}$, after integrating $\mathcal{O}_{Q_{L 1}}$ and $\mathcal{O}_{d_{R}}$, we expect the most general form

$$
\mathcal{L}_{\mathrm{bil}}^{(1)}=\frac{1}{\Lambda_{d}^{d_{H}-1}}\left(\epsilon_{b_{L}}^{(1)} \bar{Q}_{L 3}+\epsilon_{s_{L}}^{(1)} \bar{Q}_{L 2}+\epsilon_{d_{L}}^{(1)} \bar{Q}_{L 1}\right) \mathcal{O}_{H}\left(\epsilon_{b_{R}}^{(1)} b_{R}+\epsilon_{s_{R}}^{(1)} s_{R}+\epsilon_{d_{R}}^{(1)} d_{R}\right)
$$

Now, at $\Lambda_{\text {IR }}$ we identify the matrix elements of $\mathcal{O}_{H}$ with those of the SM Higgs $H$, which implies the replacement ${ }^{3}$

$$
\mathcal{O}_{H} \rightarrow g_{*} \Lambda_{\mathrm{IR}}^{d_{H}-1} H
$$

\footnotetext{
${ }^{3}$ For simplicity we are assuming a single coupling $g_{*}$, but in principle the couplings at the scales $\Lambda_{f}$ could be different.
} 
in eq. (2.2), eq. (2.4) and eq. (2.5). Then, for the down sector, we have the following "onion" Yukawa structure

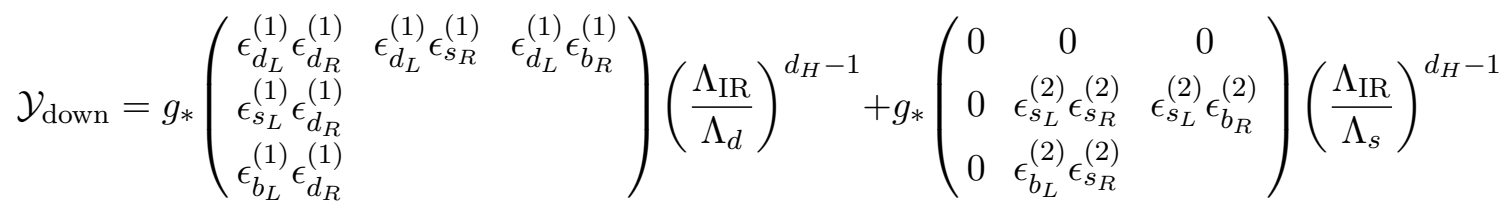

$$
\begin{aligned}
& +g_{*}\left(\begin{array}{ccc}
0 & 0 & 0 \\
0 & 0 & 0 \\
0 & 0 & \epsilon_{b_{L}}^{(3)} \epsilon_{b_{R}}^{(3)}
\end{array}\right)\left(\frac{\Lambda_{\mathrm{IR}}}{\Lambda_{b}}\right)^{d_{H}-1},
\end{aligned}
$$

where the entries that are not shown are terms that can be neglected in the limit in which we take $\Lambda_{d} \gg \Lambda_{s} \gg \Lambda_{b}$. Eq. (2.7) leads to the approximate down Yukawa matrix

$$
\mathcal{Y}_{\text {down }} \simeq\left(\begin{array}{rrr}
Y_{d} & \alpha_{R}^{d s} Y_{d} & \alpha_{R}^{d b} Y_{d} \\
\alpha_{L}^{d s} Y_{d} & Y_{s} & \alpha_{R}^{s b} Y_{s} \\
\alpha_{L}^{d b} Y_{d} & \alpha_{L}^{s b} Y_{s} & Y_{b}
\end{array}\right),
$$

where

$$
Y_{f} \equiv g_{*} \epsilon_{f_{L i}}^{(i)} \epsilon_{f_{R i}}^{(i)}\left(\frac{\Lambda_{\mathrm{IR}}}{\Lambda_{f}}\right)^{d_{H}-1}
$$

are approximately the SM Yukawas $Y_{f} \simeq m_{f} / v$. The $\alpha_{L}$ and $\alpha_{R}$ in eq. (2.8) are ratios of epsilons:

$$
\alpha_{L}^{d s} \sim \epsilon_{s_{L}}^{(1)} / \epsilon_{d_{L}}^{(1)}, \quad \alpha_{L}^{d b} \sim \epsilon_{b_{L}}^{(1)} / \epsilon_{d_{L}}^{(1)}, \quad \alpha_{L}^{s b} \sim \epsilon_{b_{L}}^{(2)} / \epsilon_{s_{L}}^{(2)},
$$

where $L \rightarrow R$ gives us the $\alpha_{R}$. Taking the largest values $\epsilon_{f_{L i, R i}}^{(i)} \sim 1$ and $g_{*} \sim 4 \pi$, we can obtain from eq. (2.9) the largest values of $\Lambda_{f}$ that allow to reproduce the SM fermion masses as a function of $d_{H}$, that we show in figure 2. For the particular case $d_{H}=2$, we have

$$
\Lambda_{f} \sim \frac{g_{*}}{Y_{f}} \Lambda_{\mathrm{IR}}
$$

that, for $\Lambda_{\mathrm{IR}} \sim 3 \mathrm{TeV}$ and $g_{*} \sim 4 \pi$, gives

$$
\Lambda_{d} \sim 3 \times 10^{9} \mathrm{GeV}, \quad \Lambda_{s} \sim 10^{8} \mathrm{GeV}, \quad \Lambda_{b} \sim 3 \times 10^{6} \mathrm{GeV} .
$$

Eq. (2.8) can be diagonalized by unitary matrices whose structure is approximately

$$
V_{L}^{\text {down }} \sim\left(\begin{array}{ccc}
1 & \alpha_{R}^{d s} \frac{Y_{d}}{Y_{s}} & \alpha_{R}^{d b} \frac{Y_{d}}{Y_{b}} \\
& 1 & \alpha_{R}^{s b} \frac{Y_{s}}{Y_{b}} \\
& & 1
\end{array}\right), \quad V_{R}^{\text {down }} \sim\left(\begin{array}{ccc}
1 & \alpha_{L}^{d s} \frac{Y_{d}}{Y_{s}} & \alpha_{L}^{d b} \frac{Y_{d}}{Y_{b}} \\
& 1 & \alpha_{L}^{s b} \frac{Y_{s}}{Y_{b}} \\
& & 1
\end{array}\right)
$$

where we omit some $i j$-entries as they are of similar size as their transpose $j i$-entries.

We can proceed in a similar way for the up sector. The large Yukawa coupling of the top implies that this must arise at $\Lambda_{\mathrm{IR}}$ as in the anarchic case, so we associate $\Lambda_{t} \sim \Lambda_{\mathrm{IR}}$. 


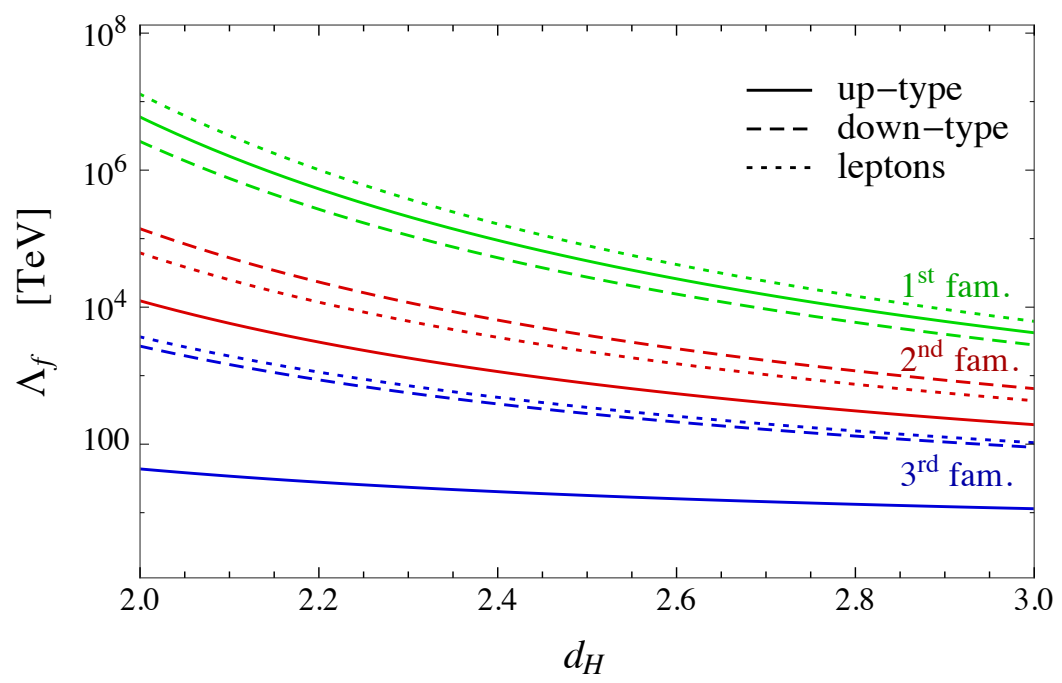

Figure 2. Upper bound on the scale $\Lambda_{f}$ (for $f=e, u, d, s, \mu, c, \tau, b, t$ from top to down) at which the fermion Yukawas can originate from a bilinear term (eq. (2.9) with $\epsilon_{f_{L i, R i}}^{(i)} \sim 1, g_{*} \sim 4 \pi$ and for $\Lambda_{\mathrm{IR}}=3 \mathrm{TeV}$ ) as a function of $d_{H}$, the dimension of the Higgs composite operator $\mathcal{O}_{H}$. To derive the numerical results we identified the fermion masses with the running masses at $1 \mathrm{TeV}$ [8], neglecting the effect of running $m_{f}$ from $\mathrm{TeV}$ to $\Lambda_{f}$.

The Yukawa matrix is expected to have the structure

$$
\mathcal{Y}_{\mathrm{up}} \simeq\left(\begin{array}{rrr}
Y_{u} & \alpha_{R}^{u c} Y_{u} & \alpha_{R}^{u t} Y_{u} \\
\alpha_{L}^{u c} Y_{u} & Y_{c} & \alpha_{R}^{c t} Y_{c} \\
\alpha_{L}^{u t} Y_{u} & \alpha_{L}^{c t} Y_{c} & Y_{t}
\end{array}\right) .
$$

We must point out however that there can be extra contributions coming from $\Lambda_{d, s, b}$. The most important ones come from $\Lambda_{d}$ where it is possible to generate

$$
\Delta \mathcal{L}_{\mathrm{bil}}^{(1)}=\frac{1}{\Lambda_{d}^{d_{H}-1}} \epsilon_{d_{L}}^{(1)} \bar{Q}_{L 1} \tilde{\mathcal{O}}_{H}\left(\tilde{\epsilon}_{t_{R}}^{(1)} t_{R}+\tilde{\epsilon}_{c_{R}}^{(1)} c_{R}\right)
$$

that leads to contributions to the entries $\left(\mathcal{Y}_{\text {up }}\right)_{13} \sim\left(\mathcal{Y}_{\text {up }}\right)_{12} \sim Y_{d}$ that can be slightly larger than those in eq. (2.14) since $Y_{d}>Y_{u}$. We absorb these contributions in eq. (2.14) by a redefinition of $\alpha_{R}^{u c, u t}$. Similarly, $\mathcal{Y}_{\text {down }}$ can receive extra contributions from $\Lambda_{u, c, t}$. The largest expected one is from $\Lambda_{c}$ where we can have

$$
\frac{1}{\Lambda_{c}^{d_{H}-1}} \bar{Q}_{L 2} \mathcal{O}_{H} b_{R}
$$

that leads to $\left(\mathcal{Y}_{\text {down }}\right)_{23} \sim Y_{c}$ that is parametrically a factor $Y_{c} / Y_{s} \sim 10$ larger than the corresponding entry in eq. (2.8). Again, we absorb this contribution in a redefinition of $\alpha_{R}^{s b}$. We must add however that if the strong sector had an SU(3) flavor symmetry, the contributions in eq. (2.15) and eq. (2.16) would be zero, as they originate from the offdiagonal interactions in the strong sector, $\mathcal{O}_{Q_{L 1}} \tilde{\mathcal{O}}_{H} \mathcal{O}_{t_{R}, c_{R}}$ and $\mathcal{O}_{Q_{L 2}} \mathcal{O}_{H} \mathcal{O}_{b_{R}}$ respectively. 
Since the mass hierarchies in the up sector are larger than in the down sector, we have that the CKM matrix $V_{\mathrm{CKM}}$ is mainly dominated by the down rotation:

$$
V_{\mathrm{CKM}} \sim\left(V_{L}^{\text {down }}\right)^{\dagger},
$$

impliying the following conditions on the $\alpha_{L}$ 's of the down-Yukawa matrix:

$$
\alpha_{R}^{d s} \frac{m_{d}}{m_{s}} \simeq\left(V_{\mathrm{CKM}}\right)_{21} \simeq \lambda_{c}, \quad \alpha_{R}^{s b} \frac{m_{s}}{m_{b}} \simeq\left(V_{\mathrm{CKM}}\right)_{32} \simeq \lambda_{c}^{2}, \quad \alpha_{R}^{d b} \frac{m_{d}}{m_{b}} \simeq\left(V_{\mathrm{CKM}}\right)_{31} \simeq \lambda_{c}^{3},
$$

where $\lambda_{c} \simeq 0.22$ is the Cabibbo angle. From the estimate

$$
\frac{m_{d}}{m_{s}} \sim \frac{m_{s}}{m_{b}} \sim \lambda_{c}^{2},
$$

we obtain using eq. (2.18) that $\alpha_{R}^{d s, d b}$ must be slightly larger than one, in particular,

$$
\alpha_{R}^{d s} \sim \alpha_{R}^{d b} \sim 1 / \lambda_{c}, \quad \alpha_{R}^{s b} \sim 1
$$

This can be easily accommodated by having $\epsilon_{s_{R}, b_{R}}^{(1)}$ slightly smaller than one (and a suppression of eq. (2.16)). On the other hand, the $\alpha_{L}$ are not constrained at all by the CKM angles, and could even be very small if some mixings are zero. For example, this could be the case if $\epsilon_{s_{L}, b_{L}}^{(1)} \approx 0$ due to some accidental discrete symmetry at $\Lambda_{d}$, as discussed in appendix B. Notice that in the limit $\epsilon_{s_{L}, b_{L}}^{(1)} \rightarrow 0$ the rotation matrix $V_{R}^{\text {down }}$ is not anymore given by eq. (2.13) but by eq. (B.1). Nevertheless, we emphasize that the framework for flavor proposed here does not need any accidental symmetry to pass the phenomenological constraints, as we discuss below.

\section{Implications in flavor and CP-violation physics}

At each scale $\Lambda_{f}$ we have potentially new flavor-violating contributions, arising from higherdimensional operators made of SM fermions. We can estimate these effects using powercounting arguments, since no flavor symmetries are assumed in our scenarios. The most important higher-dimensional operators are 4-quark operators, that contribute to $\Delta F=2$ transitions, 2-quark-2-Higgs operators that generate $\Delta F=1$ effects, and dipole operators contributing to processes such as $\mu \rightarrow e \gamma$ or EDMs. We collect the most important experimental bounds in table 1 .

\section{$3.1 \Delta F=2$ transitions}

We start considering 4-quark operators arising at the lowest scale $\Lambda_{t} \sim \Lambda_{\mathrm{IR}}$. These are operators containing only top components, $Q_{L 3}$ and $t_{R}$, namely ${ }^{4}$

$$
\frac{Y_{t}^{2} x_{t}^{2}}{\Lambda_{\mathrm{IR}}^{2}}\left(\bar{Q}_{L 3} \gamma^{\mu} Q_{L 3}\right)^{2}, \quad \frac{Y_{t}^{2}}{\Lambda_{\mathrm{IR}}^{2}}\left(\bar{Q}_{L 3} t_{R}\right)\left(\bar{t}_{R} Q_{L 3}\right), \quad \frac{Y_{t}^{2} / x_{t}^{2}}{\Lambda_{\mathrm{IR}}^{2}}\left(\bar{t}_{R} \gamma^{\mu} t_{R}\right)^{2},
$$

where we defined $x_{t}=\epsilon_{t_{L}}^{(3)} / \epsilon_{t_{R}}^{(3)}$.

\footnotetext{
${ }^{4}$ These estimates are valid even if $\Lambda_{t}>\Lambda_{\mathrm{IR}}$ and the top partners are heavier than $\Lambda_{\mathrm{IR}}$. Nevertheless, for top partners lighter than $\Lambda_{\mathrm{IR}}$, as could be needed in these scenarios to obtain a viable Higgs mass and minimize the amount of tuning [39-43], the 4-fermion operators get enhanced. For a discussion see ref. [17].
} 


\begin{tabular}{|c|c|c|c|c|}
\hline Observable & Operator & Re part & Im part & Reference \\
\hline$\Delta M_{K} ; \epsilon_{K}$ & $\begin{array}{c}\mathcal{Q}_{1}^{s d}=\left(\bar{s}_{L} \gamma^{\mu} d_{L}\right)^{2} \\
\mathcal{Q}_{2}^{s d}=\left(\bar{s}_{R} d_{L}\right)^{2}, \widetilde{\mathcal{Q}}_{2}^{s d}=\left(\bar{s}_{L} d_{R}\right)^{2} \\
\mathcal{Q}_{4}^{s d}=\left(\bar{s}_{R} d_{L}\right)\left(\bar{s}_{L} d_{R}\right)\end{array}$ & $\begin{array}{l}1.1 \times 10^{3} \\
7.3 \times 10^{3} \\
1.2 \times 10^{4}\end{array}$ & $\begin{array}{l}1.7 \times 10^{4} \\
1.2 \times 10^{5} \\
2.0 \times 10^{5}\end{array}$ & {$[22,44]$} \\
\hline$\Delta M_{B_{d}} ; S_{\psi K_{S}}$ & $\begin{array}{c}\mathcal{Q}_{1}^{b d}=\left(\bar{b}_{L} \gamma^{\mu} d_{L}\right)^{2} \\
\mathcal{Q}_{2}^{b d}=\left(\bar{b}_{R} d_{L}\right)^{2}, \widetilde{\mathcal{Q}}_{2}^{b d}=\left(\bar{b}_{L} d_{R}\right)^{2} \\
\mathcal{Q}_{4}^{b d}=\left(\bar{b}_{R} d_{L}\right)\left(\bar{b}_{L} d_{R}\right)\end{array}$ & $\begin{array}{l}6.6 \times 10^{2} \\
1.2 \times 10^{3} \\
1.6 \times 10^{3}\end{array}$ & $\begin{array}{l}9.5 \times 10^{2} \\
1.7 \times 10^{3} \\
2.3 \times 10^{3}\end{array}$ & {$[22,44]$} \\
\hline$\Delta M_{B_{s}} ; S_{\psi \phi}$ & $\begin{array}{c}\mathcal{Q}_{1}^{b s}=\left(\bar{b}_{L} \gamma^{\mu} s_{L}\right)^{2} \\
\mathcal{Q}_{2}^{b s}=\left(\bar{b}_{R} s_{L}\right)^{2}, \widetilde{\mathcal{Q}}_{2}^{b s}=\left(\bar{b}_{L} s_{R}\right)^{2} \\
\mathcal{Q}_{4}^{b s}=\left(\bar{b}_{R} s_{L}\right)\left(\bar{b}_{L} s_{R}\right)\end{array}$ & $\begin{array}{l}1.4 \times 10^{2} \\
1.3 \times 10^{2} \\
3.4 \times 10^{2}\end{array}$ & $\begin{array}{l}2.4 \times 10^{2} \\
2.2 \times 10^{2} \\
5.9 \times 10^{2}\end{array}$ & {$[22,44]$} \\
\hline$\Delta M_{D} ;|q / p|, \phi_{D}$ & $\begin{array}{c}\mathcal{Q}_{1}^{c u}=\left(\bar{c}_{L} \gamma^{\mu} u_{L}\right)^{2} \\
\mathcal{Q}_{2}^{c u}=\left(\bar{c}_{R} u_{L}\right)^{2}, \widetilde{\mathcal{Q}}_{2}^{c u}=\left(\bar{c}_{L} u_{R}\right)^{2} \\
\mathcal{Q}_{4}^{c u}=\left(\bar{c}_{R} u_{L}\right)\left(\bar{c}_{L} u_{R}\right)\end{array}$ & $\begin{array}{l}1.3 \times 10^{3} \\
2.5 \times 10^{3} \\
4.2 \times 10^{3}\end{array}$ & $\begin{array}{l}3.2 \times 10^{3} \\
5.8 \times 10^{3} \\
9.5 \times 10^{3}\end{array}$ & {$[22,44]$} \\
\hline & $\left(\bar{Q}_{L i}\left(\mathcal{Y}_{\mathrm{up}} \mathcal{Y}_{\mathrm{up}}^{\dagger}\right)_{i j} \gamma^{\mu} Q_{L j}\right)^{2}$ & \multicolumn{2}{|c|}{5} & {$[44]$} \\
\hline$b \rightarrow s \ell^{+} \ell^{-}$ & $\left(\bar{s}_{L} \gamma^{\mu} b_{L}\right) H^{\dagger} i \overleftrightarrow{D}_{\mu} H$ & 23 & 16 & {$[45,46]$} \\
\hline $\begin{array}{c}K_{L} \rightarrow \mu^{+} \mu^{-}, \\
K^{+} \rightarrow \pi^{+} \nu \bar{\nu}, \epsilon^{\prime} / \epsilon\end{array}$ & $\left(\bar{s}_{L} \gamma^{\mu} d_{L}\right) H^{\dagger} i \overleftrightarrow{D}_{\mu} H$ & \multicolumn{2}{|c|}{225} & {$[15,47]$} \\
\hline$Z \rightarrow b \bar{b}$ & $\left(\bar{b}_{L} \gamma^{\mu} b_{L}\right) H^{\dagger} i \overleftrightarrow{D}_{\mu} H$ & \multicolumn{2}{|c|}{5.5} & $\left|\delta g_{b_{L}}\right| \lesssim 10^{-3}[48,49]$ \\
\hline$B \rightarrow X_{s} \gamma$ & $\begin{array}{c}m_{b} \bar{s}_{L} \sigma^{\mu \nu} e F_{\mu \nu} b_{R} \\
m_{b} \bar{s}_{R} \sigma^{\mu \nu} e F_{\mu \nu} b_{L} \\
m_{b} \bar{s}_{L} \sigma^{\mu \nu} g_{s} G_{\mu \nu} b_{R} \\
m_{b} \bar{s}_{R} \sigma^{\mu \nu} g_{s} G_{\mu \nu} b_{L}\end{array}$ & 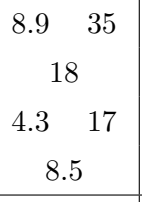 & $\begin{array}{l}18 \\
16 \\
8.6 \\
8.5 \\
\end{array}$ & {$[16]$} \\
\hline$B \rightarrow X_{d} \gamma$ & $\begin{array}{c}m_{b} \bar{d}_{L} \sigma^{\mu \nu} e F_{\mu \nu} b_{R} \\
m_{b} \bar{d}_{R} \sigma^{\mu \nu} e F_{\mu \nu} b_{L} \\
m_{b} \bar{d}_{L} \sigma^{\mu \nu} g_{s} G_{\mu \nu} b_{R} \\
m_{b} \bar{d}_{R} \sigma^{\mu \nu} g_{s} G_{\mu \nu} b_{L}\end{array}$ & $\begin{array}{l}47 \quad 19 \\
30 \\
22 \quad 9 \\
14\end{array}$ & $\begin{array}{l}37 \quad 24 \\
30 \\
18 \quad 12 \\
14\end{array}$ & {$[16]$} \\
\hline$K \rightarrow 2 \pi, \epsilon^{\prime} / \epsilon$ & $m_{s} \bar{s}_{L, R} \sigma^{\mu \nu} g_{s} G_{\mu \nu} d_{R, L}$ & & 35 & {$[16]$} \\
\hline$D \rightarrow K K, \pi \pi$ & $m_{c} \bar{c}_{L, R} \sigma^{\mu \nu} g_{s} G_{\mu \nu} u_{R, L}$ & & 27 & {$[16]$} \\
\hline Neutron EDM & $\begin{array}{c}m_{d} \bar{d}_{L} \sigma^{\mu \nu} e F_{\mu \nu} d_{R} \\
m_{u} \bar{u}_{L} \sigma^{\mu \nu} e F_{\mu \nu} u_{R} \\
m_{d} \bar{d}_{L} \sigma^{\mu \nu} g_{s} G_{\mu \nu} d_{R} \\
m_{u} \bar{u}_{L} \sigma^{\mu \nu} g_{s} G_{\mu \nu} u_{R} \\
m_{c} \bar{c}_{L} \sigma^{\mu \nu} g_{s} G_{\mu \nu} c_{R} \\
m_{b} \bar{b}_{L} \sigma^{\mu \nu} g_{s} G_{\mu \nu} b_{R} \\
m_{t} \bar{t}_{L} \sigma^{\mu \nu} g_{s} G_{\mu \nu} t_{R} \\
\end{array}$ & & $\begin{array}{l}39 \\
14 \\
48 \\
18 \\
15 \\
8.4 \\
3.7 \\
\end{array}$ & $\left|d_{n}\right|<2.9 \times 10^{-26} e \mathrm{~cm}[16,50-53]$ \\
\hline Electron EDM & $m_{e} \bar{e}_{L} \sigma^{\mu \nu} e F_{\mu \nu} e_{R}$ & & 480 & $\left|d_{e}\right|<0.87 \times 10^{-28}$ e cm [50] \\
\hline $\begin{aligned} \mu & \rightarrow e \gamma \\
\tau & \rightarrow \mu \gamma \\
\tau & \rightarrow e \gamma\end{aligned}$ & $\begin{array}{l}m_{\mu} \bar{\mu} \sigma^{\mu \nu} e F_{\mu \nu} e_{R, L} \\
m_{\tau} \bar{\tau} \sigma^{\mu \nu} e F_{\mu \nu} \mu_{R, L} \\
m_{\tau} \bar{\tau} \sigma^{\mu \nu} e F_{\mu \nu} e_{R, L}\end{array}$ & $\begin{array}{l}90 \\
3 \\
3\end{array}$ & & $\begin{array}{c}B R(\mu \rightarrow e \gamma)<5.7 \times 10^{-13}[50] \\
B R(\tau \rightarrow \mu \gamma)<4.4 \times 10^{-8}[50] \\
B R(\tau \rightarrow e \gamma)<3.3 \times 10^{-8}[50]\end{array}$ \\
\hline
\end{tabular}

Table 1. Experimental bounds on new physics contributions to flavor and CP-violating operators. The bounds are computed at an energy scale $\mu=1 \mathrm{TeV}$ and are expressed as constraints on the $\Lambda$ scale (in TeV units) parametrizing the coefficients of the operators as $C=1 / \Lambda^{2}$. Separate bounds for the real and imaginary part of the coefficients are given. When the bounds are highly asymmetric, separate ones are listed for a positive and a negative value of the coefficient. 
Let us first look at the implications in the down sector, whose flavor constraints are the strongest. These are only coming from the first operator of eq. (3.1) that, after rotating to the physical basis using eq. $(2.17),{ }^{5}$ gives a contribution to the operators $\mathcal{Q}_{1}^{s d}, \mathcal{Q}_{1}^{b d}$ and $\mathcal{Q}_{1}^{b s}$, as defined in table 1 , with a coefficient

$$
C\left(\mathcal{Q}_{1}^{s d}\right) \simeq \frac{Y_{t}^{2} x_{t}^{2}}{\Lambda_{\mathrm{IR}}^{2}}\left[\left(V_{\mathrm{CKM}}^{\dagger}\right)_{23}\left(V_{\mathrm{CKM}}\right)_{31}\right]^{2} \simeq 10^{-7} \frac{x_{t}^{2}}{\Lambda_{\mathrm{IR}}^{2}} e^{i \theta_{\mathrm{CKM}}}
$$

where $\theta_{\mathrm{CKM}}$ denotes the complex phase appearing in the product of the CKM elements, and

$$
\frac{C\left(\mathcal{Q}_{1}^{b d}\right)}{\left[\left(V_{\mathrm{CKM}}^{\dagger}\right)_{33}\left(V_{\mathrm{CKM}}\right)_{31}\right]^{2}}=\frac{C\left(\mathcal{Q}_{1}^{b s}\right)}{\left[\left(V_{\mathrm{CKM}}^{\dagger}\right)_{33}\left(V_{\mathrm{CKM}}\right)_{32}\right]^{2}}=\frac{C\left(\mathcal{Q}_{1}^{s d}\right)}{\left[\left(V_{\mathrm{CKM}}^{\dagger}\right)_{23}\left(V_{\mathrm{CKM}}\right)_{31}\right]^{2}} .
$$

Eq. (3.3) leads to interesting consequences. It predicts no new phases in $K-\bar{K}$ and $B-\bar{B}$ mixing beyond the SM one. Furthermore, it implies that the contributions to the three observables $\epsilon_{K}, \Delta M_{B_{d}}$ and $\Delta M_{B_{s}}$ are all of the order of the present experimental sensitivity. Indeed, by looking at the constraints on $\Delta F=2$ operators reported in table 1 , we find that the three observables $\epsilon_{K}, \Delta M_{B_{d}}$ and $\Delta M_{B_{s}}$ give roughly the same bound. The correlation eq. (3.3) also arises in MFV scenarios, and a bound has been derived on the size of these effects (see table 1) that leads in our case to

$$
\Lambda_{\mathrm{IR}} \gtrsim 5 x_{t} \mathrm{TeV}
$$

For $x_{t} \sim 1 / 2$ we can accommodate eq. (3.4) for values of $\Lambda_{\mathrm{IR}}$ as low as those needed to pass EWPT, $\Lambda_{\mathrm{IR}} \gtrsim 3 \mathrm{TeV}[17,48]$. The correlations in eq. (3.3) are an interesting smoking gun for these scenarios of flavor, that could be tested in the future with a better determination of the observables. In particular, we must observe a different value of $\Delta M_{B_{d, s}}$ from the SM one, with the ratio fixed:

$$
\left.\frac{\Delta M_{B_{d}}}{\Delta M_{B_{s}}} \simeq \frac{\Delta M_{B_{d}}}{\Delta M_{B_{s}}}\right|_{\mathrm{SM}}
$$

The impact in the up sector is negligible, since the mixing angles $\left(\propto Y_{u, c} / Y_{t}\right)$ are much smaller than in the down sector. The largest effect comes from the third operator in eq. (3.1), which gives a contribution

$$
C\left(\mathcal{Q}_{4}^{c u}\right) \simeq \frac{Y_{t}^{2}}{\Lambda_{\mathrm{IR}}^{2}}\left(V_{R}^{\mathrm{up}}\right)_{32}^{*}\left(V_{L}^{\mathrm{up}}\right)_{31}\left(V_{L}^{\mathrm{up}}\right)_{32}^{*}\left(V_{R}^{\mathrm{up}}\right)_{31} \sim \frac{Y_{u}^{2} Y_{c}^{2} / Y_{t}^{2}}{\Lambda_{\mathrm{IR}}^{2}} \simeq 10^{-15} \frac{1}{\Lambda_{\mathrm{IR}}^{2}},
$$

where we have taken $\alpha_{L, R} \sim 1$. This is many orders of magnitude below the experimental bound for $\Lambda_{\mathrm{IR}} \sim \mathrm{TeV}$.

Let us now move to the effects at the scales $\Lambda_{f} \gg \Lambda_{\mathrm{IR}}$. It is clear that contributions at $\Lambda_{b}$ are smaller than those of eq. (3.1), as they are suppressed by a larger scale $\Lambda_{b} \gg \Lambda_{\mathrm{IR}}$. Contributions from $\Lambda_{c}$ and $\Lambda_{s}$ can however be sizable as they involve second family quarks.

\footnotetext{
${ }^{5}$ In an abuse of notation we will be using the same notation for the quarks in the physical and interaction basis.
} 
The most relevant contributions are ${ }^{6}$

$$
\frac{g_{*}^{2} \epsilon_{c_{L}}^{(2) 4}}{\Lambda_{c}^{2}}\left(\bar{Q}_{L 2} \gamma^{\mu} Q_{L 2}\right)^{2}, \quad \frac{g_{*}^{2} \epsilon_{c_{L}}^{(2) 3} \epsilon_{t_{L}}^{(2)}}{\Lambda_{c}^{2}}\left(\bar{Q}_{L 2} \gamma^{\mu} Q_{L 3}\right)\left(\bar{Q}_{L 2} \gamma_{\mu} Q_{L 2}\right), \quad \frac{g_{*}^{2}\left(\epsilon_{s_{L}}^{(2)} \epsilon_{s_{R}}^{(2)}\right)^{2}}{\Lambda_{s}^{2}}\left(\bar{Q}_{L 2} s_{R}\right)\left(\bar{s}_{R} Q_{L 2}\right)
$$

Using eq. (2.9), we can trade the scales $\Lambda_{c, s}$ by $\Lambda_{\mathrm{IR}}$, and write eq. (3.7) for $d_{H}=2$ as

$$
\frac{Y_{c}^{2} x_{c}^{2}}{\Lambda_{\mathrm{IR}}^{2}}\left(\bar{Q}_{L 2} \gamma^{\mu} Q_{L 2}\right)^{2}, \quad \frac{Y_{c}^{2} x_{c}^{2} \alpha_{L}^{c t}}{\Lambda_{\mathrm{IR}}^{2}}\left(\bar{Q}_{L 2} \gamma^{\mu} Q_{L 3}\right)\left(\bar{Q}_{L 2} \gamma_{\mu} Q_{L 2}\right), \quad \frac{Y_{s}^{2}}{\Lambda_{\mathrm{IR}}^{2}}\left(\bar{Q}_{L 2} s_{R}\right)\left(\bar{s}_{R} Q_{L 2}\right),
$$

where $x_{c}=\epsilon_{c_{L}}^{(2)} / \epsilon_{c_{R}}^{(2)}$. After rotating to the physical basis, the operators in eq. (3.8) give respectively

$$
\begin{aligned}
& C\left(\mathcal{Q}_{1}^{s d}\right) \simeq \frac{Y_{c}^{2} x_{c}^{2}}{\Lambda_{\mathrm{IR}}^{2}}\left[\left(V_{\mathrm{CKM}}^{\dagger}\right)_{22}\left(V_{\mathrm{CKM}}\right)_{21}\right]^{2} \simeq 4 \times 10^{-7} \frac{x_{c}^{2}}{\Lambda_{\mathrm{IR}}^{2}}, \\
& C\left(\mathcal{Q}_{1}^{s d}\right) \simeq \frac{Y_{c}^{2} x_{c}^{2} \alpha_{L}^{c t}}{\Lambda_{\mathrm{IR}}^{2}}\left(V_{\mathrm{CKM}}^{\dagger}\right)_{22}^{2}\left(V_{\mathrm{CKM}}\right)_{21}\left(V_{\mathrm{CKM}}\right)_{31} \simeq 1.6 \times 10^{-8} \frac{x_{c}^{2} \alpha_{L}^{c t}}{\Lambda_{\mathrm{IR}}^{2}}, \\
& C\left(\mathcal{Q}_{4}^{s d}\right) \simeq \frac{Y_{s}^{2}}{\Lambda_{\mathrm{IR}}^{2}}\left(V_{\mathrm{CKM}}^{\dagger}\right)_{22}\left(V_{R}^{\text {down }}\right)_{21}\left(V_{R}^{\text {down }}\right)_{22}^{*}\left(V_{\mathrm{CKM}}\right)_{21} \simeq 9 \times 10^{-10} \frac{\alpha_{L}^{d s}}{\Lambda_{\mathrm{IR}}^{2}} .
\end{aligned}
$$

The first contribution is real and therefore only affects $\Delta M_{K}$, while the other two can be complex and contribute to $\epsilon_{K}$. Their experimental bounds lead to ${ }^{7}$

$$
\Lambda_{\mathrm{IR}} \gtrsim 0.6 x_{c} \mathrm{TeV}, \quad \Lambda_{\mathrm{IR}} \gtrsim 1.8 x_{c} \sqrt{\alpha_{L}^{c t}} \mathrm{TeV}, \quad \Lambda_{\mathrm{IR}} \gtrsim 5 \sqrt{\alpha_{L}^{d s}} \mathrm{TeV} .
$$

To derive these bounds we have assumed that the contributions eq. (3.10) and eq. (3.11) have maximal complex phase $\sim \pi / 4$, as we will assume throughout the article. The bounds in eq. (3.12) are roughly comparable to the one in eq. (3.4), and can be accommodated for $\Lambda_{\mathrm{IR}}$ of $f e w \mathrm{TeV}$. These extra contributions to $\epsilon_{K}$ spoil the correlation in eq. (3.3), but preserve eq. (3.5). Indeed, it is easy to realize that contributions at $\Lambda_{c, s}$ to $B$ physics (and also $D$ physics) are negligible.

Finally, we also have contributions arising at $\Lambda_{d}$. The most relevant ones are those to the operator $\mathcal{Q}_{4}^{s d}$. For $d_{H}=2$ we have

$$
C\left(\mathcal{Q}_{4}^{s d}\right) \simeq \frac{Y_{d}^{2} \alpha_{L}^{d s} \alpha_{R}^{d s}}{\Lambda_{\mathrm{IR}}^{2}} \simeq 9 \times 10^{-10} \frac{\alpha_{L}^{d s}}{\Lambda_{\mathrm{IR}}^{2}},
$$

where we have used eq. (2.20). This contributions are as sizable as eq. (3.11).

The above conclusions however drastically depend on $d_{H}$. For $d_{H}>2$ we have that the contributions from $\Lambda_{c, s, d}$ are enhanced, with respect to eqs. (3.9)-(3.11) and eq. (3.13),

\footnotetext{
${ }^{6}$ Notice that contributions to the $\mathcal{Q}_{2}$ and $\widetilde{\mathcal{Q}}_{2}$ operators require two Higgs insertions and are thus highly suppressed.

${ }^{7}$ In computing the bounds on operators generated at $\Lambda_{f} \gg \Lambda_{\mathrm{IR}}$, running effects should also be taken into account. These include the running of $Y_{f}$ (which decrease at high energy), as well as the running of the 4 -fermion effective interactions (which determine a mild increase in the bounds for the $\mathcal{Q}_{4}$ operators). These two effects partially compensate each other. Since the numerical impact is not large, for simplicity we will not take into account the running in our estimates.
} 
by a factor $\left(\Lambda_{f} / \Lambda_{\mathrm{IR}}\right)^{2 d_{H}-4}$. Therefore $d_{H}>2$ can only be consistent with the experimental bounds if $\Lambda_{f} \sim \Lambda_{\mathrm{IR}}$ that corresponds to the anarchic scenario. This implies that generating the mass for the charm, strange or down from bilinear mixing at $\Lambda_{f} \gg \Lambda_{\mathrm{IR}}$ is only possible for $d_{H} \lesssim 2$.

\section{$3.2 \quad$ Neutron EDM}

Dipole operators can also be induced at $\Lambda_{f}$. These operators are strongly constrained, in particular from the measurement of the neutron EDM, which place a bound on quark dipole operators of the form

$$
c_{\mathrm{edm}}^{q} \bar{q}_{L} \sigma^{\mu \nu} g_{s} G_{\mu \nu} q_{R},
$$

or analogous operators involving the photon field-strength (see table 1). In the anarchic case the current measurements lead to very severe bounds, $\Lambda_{\mathrm{IR}} \gtrsim 48\left(g_{*} / 4 \pi\right) \mathrm{TeV}$ from the down-quark EDM, and $\Lambda_{\mathrm{IR}} \gtrsim 18\left(g_{*} / 4 \pi\right) \mathrm{TeV}$ from the up-quark EDM. These bounds were calculated under the assumption that dipole operators are induced at the one-loop level and therefore must carry a factor $g_{*}^{2} / 16 \pi^{2}$ [8], as it occurs in holographic descriptions of the model [7]. Obviously, for maximal coupling $g_{*} \sim 4 \pi$ this loop factor is of order one, not introducing any extra suppression. Hereafter we will also follow this assumption for our estimates.

In our scenarios for flavor the contributions to $c_{\text {edm }}^{u, d}$ are all very small, due to either small mixings or a large scale $\Lambda_{f}$ suppressing the processes. In fact, the main contribution to the neutron EDM comes from a top EDM that can be induced at $\Lambda_{\mathrm{IR}}$ with a size

$$
c_{\mathrm{edm}}^{t} \simeq \frac{g_{*}^{2}}{16 \pi^{2}} \frac{m_{t}}{\Lambda_{\mathrm{IR}}^{2}} .
$$

According to the bound in table 1 , we obtain $\Lambda_{\mathrm{IR}} \gtrsim 3\left(g_{*} / 4 \pi\right) \mathrm{TeV}$, implying that we expect in these scenarios a neutron EDM below, but not much smaller than, its present experimental limit.

Contributions originating at $\Lambda_{f}$ are much smaller. The reason is that EDM operators must involve the Higgs field that at high energies corresponds to the composite operator $\mathcal{O}_{H}$ of dimension larger than one. Therefore the contribution to EDMs is suppressed by $d_{H}+1$ powers of $\Lambda_{f}$. For example, at $\Lambda_{b}$, we expect a bottom-EDM from the operator

$$
\frac{g_{*}^{2}}{16 \pi^{2}} \frac{\epsilon_{b_{L}}^{(3)} \epsilon_{b_{R}}^{(3)}}{\Lambda_{b}^{d_{H}+1}} \bar{Q}_{L 3} \mathcal{O}_{H} \sigma^{\mu \nu} g_{s} G_{\mu \nu} b_{R},
$$

which gives

$$
c_{\mathrm{edm}}^{b} \simeq \frac{g_{*}^{2}}{16 \pi^{2}} \frac{m_{b}}{\Lambda_{b}^{2}} .
$$

This is much smaller than present bounds unless $\Lambda_{b} \sim \Lambda_{\mathrm{IR}}$.

\section{3 $\Delta F=1$ transitions}

Similarly to EDMs, contributions to flavor dipole transitions can also be present, the most relevant ones being $\bar{s}_{R, L} \sigma^{\mu \nu} e F_{\mu \nu} b_{L, R}$ that contributes to $b \rightarrow s \gamma$, and $\bar{s}_{R, L} \sigma^{\mu \nu} g_{s} G_{\mu \nu} d_{L, R}$ 
that contributes to $\epsilon^{\prime} / \epsilon$. The estimates of these effects are similar to the ones for the neutron EDM in eq. (3.16), leading to small contributions to these observables.

There are also non-dipole contributions to $\Delta F=1$ transitions arising from operators like $\bar{s}_{L} \gamma^{\mu} d_{L} H^{\dagger} \overleftrightarrow{D}_{\mu} H$ that on the EWSB vacuum give flavor-changing $Z$-couplings, which are severely constrained by $K_{L} \rightarrow \mu^{+} \mu^{-}$and $\epsilon^{\prime} / \epsilon$, or equivalent operators with the bottom, $\bar{s}_{L} \gamma_{\mu} b_{L} H^{\dagger} \overleftrightarrow{D}_{\mu} H$, which give contributions to the processes $B \rightarrow \ell^{+} \ell^{-}, X \ell^{+} \ell^{-}$. The largest contribution arises from top operators induced at $\Lambda_{\mathrm{IR}}$ that give

$$
\begin{aligned}
\frac{\left(g_{*} \epsilon_{t_{L}}^{(3)}\right)^{2}}{\Lambda_{\mathrm{IR}}^{2}} \bar{Q}_{L 3} \gamma^{\mu} Q_{L 3} i H^{\dagger} \overleftrightarrow{D}_{\mu} H \simeq & \frac{g_{*} Y_{t} x_{t}}{\Lambda_{\mathrm{IR}}^{2}}\left(\left(V_{\mathrm{CKM}}^{\dagger}\right)_{33} \bar{b}_{L}+\left(V_{\mathrm{CKM}}^{\dagger}\right)_{23} \bar{s}_{L}+\left(V_{\mathrm{CKM}}^{\dagger}\right)_{13} \bar{d}_{L}\right) \gamma^{\mu} \\
& \times\left(\left(V_{\mathrm{CKM}}\right)_{33} b_{L}+\left(V_{\mathrm{CKM}}\right)_{32} s_{L}+\left(V_{\mathrm{CKM}}\right)_{31} d_{L}\right) i H^{\dagger} \overleftrightarrow{D}_{\mu} H,
\end{aligned}
$$

similarly to the anarchic case. Interestingly, eq. (3.18) shows that the contributions to $K_{L} \rightarrow \mu^{+} \mu^{-}$(and $\epsilon^{\prime} / \epsilon$ ), $B \rightarrow(X) \ell \ell$ and corrections to $Z \bar{b}_{L} b_{L}$ are correlated and all are close to the experimental bounds; we obtain respectively the constraints

$$
\Lambda_{\mathrm{IR}} \gtrsim 4 \sqrt{g_{*} x_{t}} \mathrm{TeV}, \quad \Lambda_{\mathrm{IR}} \gtrsim 3 \sqrt{g_{*} x_{t}} \mathrm{TeV}, \quad \Lambda_{\mathrm{IR}} \gtrsim 5 \sqrt{g_{*} x_{t}} \mathrm{TeV} .
$$

We must point out however that there is another dimension-six operator contributing to these observables, $\bar{Q}_{L 3} \sigma^{a} \gamma^{\mu} Q_{L 3} H^{\dagger} \sigma^{a} \overleftrightarrow{D}_{\mu} H$, that in the case of a custodial $P_{L R}$ symmetry in the strong sector cancels the contribution from eq. (3.18) [54]. This symmetry is present in simple models of composite Higgs and for this reason these effects could be further suppressed.

Finally, there can be also contributions to operators like $\bar{s}_{L} \gamma_{\mu} d_{L} D_{\nu} F_{Z}^{\mu \nu}$, where $F_{Z}^{\mu \nu}$ is the field-strength of the $Z$. These operators, however, are suppressed by a factor $g^{2} / g_{*}^{2}$ with respect to those in eq. (3.18).

\subsection{Electron EDM, $\mu \rightarrow e \gamma$ and $\tau \rightarrow \mu \gamma$}

Assuming that the origin of the lepton masses is the same one as for the down-type quark masses described above, we expect $\mathcal{Y}_{\text {lepton }}$ and the rotation matrices to have the same structure as eq. (2.8) and eq. (2.13) respectively, with the obvious replacement $d, s, b \rightarrow$ $e, \mu, \tau$. The corresponding $\alpha_{L, R}$ for the lepton sector are free parameters, that we will take to be order one for our estimates.

The main experimental constraints on possible effective operators induced at the scales $\Lambda_{e, \mu, \tau}$ are the electron EDM, $\mu \rightarrow e \gamma$ and $\tau \rightarrow \mu \gamma$, that come from similar dipole structures:

$$
c_{\mathrm{edm}}^{e} \bar{e}_{L} \sigma^{\mu \nu} e F_{\mu \nu} e_{R}, \quad c_{\mathrm{meg}} \bar{e}_{L} \sigma^{\mu \nu} e F_{\mu \nu} \mu_{R}, \quad c_{\mathrm{tmg}} \bar{\mu}_{L} \sigma^{\mu \nu} e F_{\mu \nu} \tau_{R}
$$

and analogous ones obtained interchanging the chiralities, $L \leftrightarrow R$. In the anarchic case the first two operators in eq. (3.20) put the most severe constraints (see table 3). In our scenarios, however, we find that these contributions are very small for the same reason as for the neutron EDM. The largest contribution arises at $\Lambda_{\tau}$, and give for $d_{H}=2$

$$
c_{\mathrm{edm}}^{e} \simeq\left(\frac{g_{*}}{4 \pi}\right)^{2}\left(V_{L}^{\text {lepton }}\right)_{31}^{*}\left(V_{R}^{\text {lepton }}\right)_{31} \frac{g_{*} v \Lambda_{\mathrm{IR}}}{\Lambda_{\tau}^{3}} \sim\left(\frac{g_{*}}{4 \pi}\right)^{2} \frac{Y_{e} Y_{\tau}}{g_{*}^{2}} \frac{m_{e}}{\Lambda_{\mathrm{IR}}^{2}}
$$




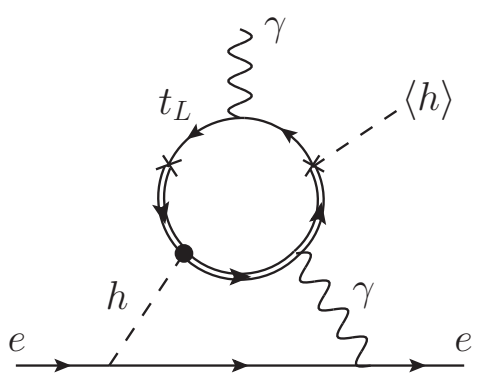

Figure 3. A representative two-loop contribution to the electron EDM. The double-line represents a resonance from the strong sector.

which is extremely small. Similarly, for $\mu \rightarrow e \gamma$ and $\tau \rightarrow \mu \gamma$, we get at $\Lambda_{\tau}$ :

$$
\begin{aligned}
& c_{\mathrm{meg}} \simeq\left(\frac{g_{*}}{4 \pi}\right)^{2}\left(V_{L}^{\text {lepton }}\right)_{32}^{*}\left(V_{R}^{\text {lepton }}\right)_{31} \frac{g_{*} v \Lambda_{\mathrm{IR}}}{\Lambda_{\tau}^{3}} \sim\left(\frac{g_{*}}{4 \pi}\right)^{2} \frac{Y_{e} Y_{\tau}}{g_{*}^{2}} \frac{m_{\mu}}{\Lambda_{\mathrm{IR}}^{2}}, \\
& c_{\mathrm{tmg}} \simeq\left(\frac{g_{*}}{4 \pi}\right)^{2}\left(V_{L}^{\text {lepton }}\right)_{32}^{*} \frac{g_{*} v \Lambda_{\mathrm{IR}}}{\Lambda_{\tau}^{3}} \sim\left(\frac{g_{*}}{4 \pi}\right)^{2} \frac{Y_{\mu} Y_{\tau}}{g_{*}^{2}} \frac{m_{\tau}}{\Lambda_{\mathrm{IR}}^{2}},
\end{aligned}
$$

that are several orders of magnitude below the experimental bound.

Additional contributions to the electron EDM can come from Barr-Zee-type 2-loop diagrams [33] as shown in figure 3. These involve CP-violating one-loop induced vertices such as $H^{\dagger} D_{\rho}^{2} H \tilde{F}_{\mu \nu} F^{\mu \nu}$ arising from the strong sector, mainly from a loop of top resonances. ${ }^{8}$ The estimate of the size of these couplings are very model dependent. In the particular motivated case of a pseudo-Nambu-Goldstone boson (PNGB) Higgs these couplings cannot be generated from the strong sector alone, as they are protected by the global symmetry under which the Goldstone Higgs transforms. Therefore we need a SM particle to be involved in the loop. We can take as an estimate the contribution involving the $t_{L}$ (see figure 3) that induces the vertex $H^{\dagger} D_{\rho}^{2} H \tilde{F}_{\mu \nu} F^{\mu \nu}$ with a coefficient $\sim e^{2} x_{t} Y_{t} g_{*} /\left(16 \pi^{2}\right)$ (omitting powers of $\Lambda_{\mathrm{IR}}$ ). Using the results of ref. [55], in which the Barr-Zee contribution to the electron EDM is computed in the presence of CP-violating Higgs interactions to the top, $-i \widetilde{\kappa}_{t} Y_{t}\left(\bar{t} \gamma_{5} t\right) h / \sqrt{2}$, and found $\left|\widetilde{\kappa}_{t}\right|<0.01$, we have, after the proper rescaling for our case,

$$
\frac{x_{t} Y_{t} g_{*}}{\Lambda_{\mathrm{IR}}^{2}} \lesssim 0.01 \frac{Y_{t}^{2}}{m_{t}^{2}}
$$

that leads to the bound

$$
\Lambda_{\mathrm{IR}} \gtrsim 1.6 \sqrt{g_{*} x_{t}} \mathrm{TeV} .
$$

The size of this correction is thus comparable with the present experimental bounds and should be visible in future experiments. Notice that in the cases in which the Higgs is not a PNGB, this effect is enhanced by a factor $g_{*} / Y_{t}$.

\footnotetext{
${ }^{8}$ There is also the possibility to have a vertex involving a $Z$, but this contribution to the EDM is suppressed as a consequence of $C$ invariance that makes only the (very small) vector part of the $Z$ coupling to the electron to contribute [33].
} 
Barr-Zee-type contributions to $\mu \rightarrow e \gamma$ are also sizeable in anarchic models [56], but in our scenarios for flavor these contributions are very small since the Higgs flavor-changing couplings to leptons are highly suppressed — see section 5 .

\section{Alternative scenarios}

Although so far we considered a scenario in which the different fermion masses arise at different UV scales $\Lambda_{f}$, we could also consider simpler cases with fewer UV scales or with more particles than the top with masses arising from partial compositeness at $\Lambda_{\mathrm{IR}}$. In the following we present several alternative scenarios pointing out in which cases there is a clash with the experimental bounds.

\section{- First-family masses generated at the same scale $\Lambda_{\mathbf{1}}$.}

We could take the economical assumption that all first-family fermion masses arise at the same scale $\Lambda_{1} \sim \Lambda_{d} \sim 3 \times 10^{8} \mathrm{GeV}$, corresponding to the scale of the heaviest fermion, the down quark. The fact that $m_{e}<m_{u}<m_{d}$ could be accommodated in this case by taking the mixing terms $\epsilon_{e_{R}, e_{L}}^{(1)}$ and $\epsilon_{u_{R}}^{(1)}$ to be slightly smaller than one. None of the estimates made in the previous section are changed in this case. The reason is that none of the main contributions were originating at $\Lambda_{u}$ or $\Lambda_{e}$, as these were very small.

\section{- Second-family masses generated at the same scale $\Lambda_{2}$.}

Similarly, we could assume that all second-family fermions get their masses at one single scale $\Lambda_{2} \sim \Lambda_{c} \sim 10^{6} \mathrm{GeV}$. Again, it is easy to show that the estimates of the previous section are not affected. Of course, contributions at the scale $\Lambda_{2}$ to up quark and electron EDM, as well as $\mu \rightarrow e \gamma$ are larger now as $\Lambda_{2} \gg \Lambda_{s, \mu}$, but these are still few orders of magnitude below the experimental bounds. Contributions to $\Delta F=2$ 4-fermion interactions are however not affected, since for $d_{H} \simeq 2$ they can be written, using eq. (2.9), as a function of $Y_{s}$ and $\Lambda_{\mathrm{IR}}$, independently of $\Lambda_{s}$.

\section{- Partly-composite third-family fermions at $\Lambda_{\mathrm{IR}}$.}

Following the above approach of family reunion, we can consider the case in which all third family fermions are, analogously to the top, partly composite, i.e., having their masses arising at $\Lambda_{\mathrm{IR}}$.

- Partly-composite bottom: in this case there are new contributions to $\Delta F=2$ that have the same structure as eq. (3.1) but with the replacement $t_{R} \rightarrow b_{R}$ and $Y_{t} \rightarrow Y_{b}$. Due to the $Y_{b}$ suppression, one gets contributions much smaller than the present bounds. There is also now a larger contribution to the bottom-quark EDM, arising at $\Lambda_{\mathrm{IR}}$ :

$$
c_{\mathrm{edm}}^{b} \simeq\left(\frac{g_{*}}{4 \pi}\right)^{2} \frac{m_{b}}{\Lambda_{\mathrm{IR}}^{2}},
$$

which saturates the experimental bound for $\Lambda_{\mathrm{IR}} \sim 7\left(g_{*} / 4 \pi\right) \mathrm{TeV}$. Additional contributions to the $b \rightarrow s$ and $s \rightarrow d$ transitions as well as to the $Z b \bar{b}$ coupling 
are also generated, which are slightly suppressed with respect to the ones coming from the top partial compositeness (see table 3).

- Partly-composite tau: in this case the most relevant observable is $\mu \rightarrow e \gamma$ that receives at $\Lambda_{\mathrm{IR}}$ a contribution of order

$$
\begin{aligned}
c_{\mathrm{meg}} \simeq\left(\frac{g_{*}}{4 \pi}\right)^{2} \frac{m_{\tau}}{\Lambda_{\mathrm{IR}}^{2}}\left(V_{L}^{\text {lepton }}\right)_{23}^{*}\left(V_{R}^{\text {lepton }}\right)_{13} & \simeq \alpha_{L}^{\mu \tau} \alpha_{R}^{e \tau}\left(\frac{g_{*}}{4 \pi}\right)^{2} \frac{Y_{e}}{Y_{\tau}} \frac{m_{\mu}}{\Lambda_{\mathrm{IR}}^{2}} \\
& \simeq 3 \times 10^{-4} \alpha_{L}^{\mu \tau} \alpha_{R}^{e \tau}\left(\frac{g_{*}}{4 \pi}\right)^{2} \frac{m_{\mu}}{\Lambda_{\mathrm{IR}}^{2}},
\end{aligned}
$$

and a similar contribution with $\alpha_{R} \leftrightarrow \alpha_{L}$. From eq. (4.2) and the experimental bound in table 1 , we get

$$
\Lambda_{\mathrm{IR}} \gtrsim 15 \sqrt{\alpha_{L}^{\mu \tau} \alpha_{R}^{e \tau}}\left(\frac{g_{*}}{4 \pi}\right) \mathrm{TeV},
$$

which shows that these corrections can be close to the experimental bound, motivating a better measurement of $\mu \rightarrow e \gamma$ as a probe for this scenario. Similarly, the electron EDM and $\tau \rightarrow \mu \gamma$ are also enhanced if the tau is partly composite, leading to the estimates

$$
c_{\mathrm{edm}}^{e} \simeq \frac{\alpha_{L}^{e \tau}}{\alpha_{L}^{\mu \tau}} \frac{m_{e}}{m_{\mu}} c_{\mathrm{meg}}, \quad c_{\mathrm{tmg}} \simeq \frac{1}{\alpha_{R}^{e \tau}} \frac{Y_{\mu}}{Y_{e}} \frac{m_{\tau}}{m_{\mu}} c_{\mathrm{meg}},
$$

which saturates the present experimental bounds respectively for

$$
\Lambda_{\mathrm{IR}} \gtrsim 7 \sqrt{\alpha_{L}^{e \tau} \alpha_{R}^{e \tau}}\left(\frac{g_{*}}{4 \pi}\right) \mathrm{TeV}, \quad \Lambda_{\mathrm{IR}} \gtrsim 8 \sqrt{\alpha_{L}^{\mu \tau}}\left(\frac{g_{*}}{4 \pi}\right) \mathrm{TeV} .
$$

Similar bounds apply for $\alpha_{R} \leftrightarrow \alpha_{L}$.

In summary, if all the third-family fermions are partly composite at $\Lambda_{\mathrm{IR}}$, we could in the near future see a positive result from searches for neutron and electron EDM, $\mu \rightarrow e \gamma$ or $\tau \rightarrow \mu \gamma$.

\section{- Partly-composite second-family fermions at $\Lambda_{\mathrm{IR}}$.}

As a last example, it can be instructive to consider a case where all except the firstfamily fermions get their mass from partial compositeness at $\Lambda_{\mathrm{IR}}$.

- Partly-composite charm: if the charm is partly composite, there are new contributions to $\epsilon_{K}$, but they go exactly as those in eq. (3.9). The are also larger contributions to $\Delta M_{D}$. We find that they can be a factor $Y_{t}^{2} / Y_{c}^{2} \sim 10^{5}$ larger than those in eq. (3.6), nevertheless they are still below the experimental bound. The most important new contribution arises for the charm-EDM:

$$
c_{\mathrm{edm}}^{c} \simeq\left(\frac{g_{*}}{4 \pi}\right)^{2} \frac{m_{c}}{\Lambda_{\mathrm{IR}}^{2}},
$$

which saturates the experimental bound for $\Lambda_{\mathrm{IR}} \sim 13\left(g_{*} / 4 \pi\right) \mathrm{TeV}$. 


\begin{tabular}{|c|c|c|c|c|c|}
\hline$\Delta F=2$ & $t$ partly-comp. & $s$ partly-comp. & bilin. mixing (2nd fam.) & bilin. mixing (1st fam.) & Anarchic \\
\hline $\mathcal{Q}_{1}^{s d}$ & $\Lambda_{\mathrm{IR}} \gtrsim 5 x_{t}$ & $\Lambda_{\mathrm{IR}} \gtrsim 4 x_{t}$ & $\Lambda_{\mathrm{IR}} \gtrsim 1.8 x_{c} \sqrt{\alpha_{L}^{c t}}$ & $\Lambda_{\mathrm{IR}} \gtrsim 0.2 x_{d}$ & $\Lambda_{\mathrm{IR}} \gtrsim 4 x_{t}$ \\
\hline $\mathcal{Q}_{2}^{s d}$ & - & $\Lambda_{\mathrm{IR}} \gtrsim 1 \sqrt{g_{*}}$ & & & $\Lambda_{\mathrm{IR}} \gtrsim 1 \sqrt{g_{*}}$ \\
\hline$\widetilde{\mathcal{Q}}_{2}^{s d}$ & - & $\Lambda_{\mathrm{IR}} \gtrsim 0.5 \sqrt{g_{*} \alpha_{L}^{d s}}$ & & & $\Lambda_{\mathrm{IR}} \gtrsim 1 \sqrt{g_{*}}$ \\
\hline $\mathcal{Q}_{4}^{s d}$ & - & $\Lambda_{\mathrm{IR}} \gtrsim 5 \sqrt{\alpha_{L}^{d s}}$ & $\Lambda_{\mathrm{IR}} \gtrsim 5 \sqrt{\alpha_{L}^{d s}}$ & $\Lambda_{\mathrm{IR}} \gtrsim 5 \sqrt{\alpha_{L}^{d s}}$ & $\Lambda_{\text {IR }} \gtrsim 10$ \\
\hline $\mathcal{Q}_{1}^{b d}$ & $\Lambda_{\mathrm{IR}} \gtrsim 5 x_{t}$ & $\Lambda_{\mathrm{IR}} \gtrsim 6 x_{t}$ & & & $\Lambda_{\mathrm{IR}} \gtrsim 6 x_{t}$ \\
\hline$\widetilde{\mathcal{Q}}_{2}^{b d}$ & - & $\Lambda_{\mathrm{IR}} \gtrsim 0.3 \sqrt{g_{*} \alpha_{L}^{d s}}$ & & . & $\Lambda_{\mathrm{IR}} \gtrsim 0.6 \sqrt{g_{*}}$ \\
\hline $\mathcal{Q}_{4}^{b d}$ & - & $\Lambda_{\mathrm{IR}} \gtrsim 0.4 \sqrt{\alpha_{L}^{s d}}$ & $\Lambda_{\mathrm{IR}} \gtrsim 0.3 \sqrt{\alpha_{L}^{d b}}$ & . & $\Lambda_{\mathrm{IR}} \gtrsim 0.8$ \\
\hline $\mathcal{Q}_{1}^{b s}$ & $\Lambda_{\mathrm{IR}} \gtrsim 5 x_{t}$ & $\Lambda_{\mathrm{IR}} \gtrsim 7 x_{t}$ & $\Lambda_{\mathrm{IR}} \gtrsim 0.6 \alpha_{R}^{c b} x_{c}$ & & $\Lambda_{\mathrm{IR}} \gtrsim 7 x_{t}$ \\
\hline$\widetilde{\mathcal{Q}}_{2}^{b s}$ & - & $\Lambda_{\mathrm{IR}} \gtrsim 0.4 \sqrt{g_{*}}$ & & . & $\Lambda_{\mathrm{IR}} \gtrsim 0.4 \sqrt{g_{*}}$ \\
\hline $\mathcal{Q}_{4}^{b s}$ & - & $\Lambda_{\mathrm{IR}} \gtrsim 1$ & $\Lambda_{\mathrm{IR}} \gtrsim 0.1 \sqrt{\alpha_{L}^{s b}}$ & . & $\Lambda_{\mathrm{IR}} \gtrsim 1$ \\
\hline $\mathcal{Q}_{1}^{c u}$ & & & & & $\Lambda_{\mathrm{IR}} \gtrsim 1 x_{t}$ \\
\hline $\mathcal{Q}_{2}^{c u}$ & . & & . & . & $\Lambda_{\mathrm{IR}} \gtrsim 0.7 \sqrt{g_{*}}$ \\
\hline $\mathcal{Q}_{4}^{c u}$ & & & & & $\Lambda_{\mathrm{IR}} \gtrsim 1.1$ \\
\hline
\end{tabular}

Table 2. Bounds on $\Lambda_{\mathrm{IR}}$ for the different scenarios considered in the text. The effects are separated according to their origin: from the top (or strange) partial compositeness at $\Lambda_{\mathrm{IR}}$, or from the UV scale $\Lambda_{f}$ at which the second and first families get bilinear mixings to the Higgs. The results are given in TeV. Entries with a "." correspond to negligible bounds, while "- " means that the corresponding operator is not generated. The most relevant constraints are highlighted in boldface.

- Partly-composite strange: in this scenario we find the same contribution as in the anarchic case in $K$ physics, shown in table 3. Sizable contributions to the down-quark EDM are also generated:

$$
c_{\mathrm{edm}}^{d} \simeq\left(\frac{g_{*}}{4 \pi}\right)^{2} \frac{m_{s}}{\Lambda_{\mathrm{IR}}^{2}}\left(V_{\mathrm{CKM}}\right)_{21}^{*}\left(V_{R}^{\text {down }}\right)_{12} \simeq 0.2 \alpha_{L}^{d s}\left(\frac{g_{*}}{4 \pi}\right)^{2} \frac{m_{d}}{\Lambda_{\mathrm{IR}}^{2}},
$$

which leads to the bound $\Lambda_{\mathrm{IR}} \gtrsim 19\left(g_{*} / 4 \pi\right) \sqrt{\alpha_{L}^{d s}} \mathrm{TeV}$.

- Partly-composite muon: in this case the estimate for the contribution to $\mu \rightarrow e \gamma$ and electron EDM are enhanced with respect to those to the partly-composite tau (see eq. (4.2) and eq. (4.4)) by a factor $Y_{\tau} / Y_{\mu} \sim 17$. This pushes the bound on $\Lambda_{\mathrm{IR}}$ beyond the $\mathrm{TeV}$ scale, dominantly due to $\mu \rightarrow e \gamma$.

We conclude that the option with partly-composite second family at $\Lambda_{\mathrm{IR}}$ seems disfavored by the present experimental data, mainly due to EDMs and $\mu \rightarrow e \gamma$. A summary of all bounds is presented in tables 2 and 3 .

\section{Higgs couplings to fermions}

The predictions for Higgs couplings depend on the origin of the fermions masses. Here we will present the predictions for the models of flavor considered above. We will focus on models in which the Higgs arises as a PNGB from the strong sector. These models, 


\begin{tabular}{|c|c|c|c|c|}
\hline$\Delta F=1$ & $t$ partly comp. & $b$ partly comp. & $s$ partly comp. & Anarchic \\
\hline $\begin{array}{l}\bar{s}_{L} \sigma^{\mu \nu} e F_{\mu \nu} b_{R} \\
\bar{s}_{R} \sigma^{\mu \nu} e F_{\mu \nu} b_{L}\end{array}$ & - & $\Lambda_{\mathrm{IR}} \gtrsim 0.12 g_{*}$ & $\begin{array}{l}\Lambda_{\mathrm{IR}} \gtrsim 0.12 g_{*} \\
\boldsymbol{\Lambda}_{\mathrm{IR}} \gtrsim \mathbf{0 . 8} \boldsymbol{g}_{*}\end{array}$ & $\begin{array}{l}\Lambda_{\mathrm{IR}} \gtrsim 0.12 g_{*} \\
\boldsymbol{\Lambda}_{\mathrm{IR}} \gtrsim \mathbf{0 . 8} \boldsymbol{g}_{*}\end{array}$ \\
\hline $\begin{array}{l}\bar{s}_{L} \sigma^{\mu \nu} g_{s} G_{\mu \nu} d_{R} \\
\bar{s}_{R} \sigma^{\mu \nu} g_{s} G_{\mu \nu} d_{L}\end{array}$ & - & . & $\begin{array}{c}\Lambda_{\mathrm{IR}} \gtrsim 0.5 g_{*} \\
\boldsymbol{\Lambda}_{\mathrm{IR}} \gtrsim \mathbf{1 . 1} \boldsymbol{g}_{*}\end{array}$ & $\begin{array}{l}\Lambda_{\mathrm{IR}} \gtrsim 1.1 g_{*} \\
\Lambda_{\mathrm{IR}} \gtrsim 1.1 g_{*}\end{array}$ \\
\hline $\bar{s}_{L} \gamma^{\mu} b_{L} H^{\dagger} i \overleftrightarrow{D}_{\mu} H$ & $\Lambda_{\mathrm{IR}} \gtrsim \mathbf{3} \sqrt{\boldsymbol{g}_{*} \boldsymbol{x}_{\boldsymbol{t}}}(*)$ & $\Lambda_{\mathrm{IR}} \gtrsim 0.4 \sqrt{g_{*} x_{b}}$ & $\Lambda_{\mathrm{IR}} \gtrsim 0.4 \sqrt{g_{*} x_{b}}$ & $\Lambda_{\mathrm{IR}} \gtrsim 3 \sqrt{g_{*} x_{t}}$ \\
\hline $\bar{s}_{L} \gamma^{\mu} d_{L} H^{\dagger} i \overleftrightarrow{D}_{\mu} H$ & $\Lambda_{\mathrm{IR}} \gtrsim 4 \sqrt{g_{*} x_{\boldsymbol{t}}}(*)$ & $\Lambda_{\mathrm{IR}} \gtrsim 0.50 \sqrt{g_{*} x_{b}}$ & $\Lambda_{\mathrm{IR}} \gtrsim 0.5 \sqrt{g_{*} x_{b}}$ & $\Lambda_{\mathrm{IR}} \gtrsim 4 \sqrt{g_{*} x_{t}}$ \\
\hline$\Delta F=0$ & $t$ partly-comp. & $b$ partly-comp. & $s$ partly-comp. & Anarchic \\
\hline $\bar{b}_{L} \gamma^{\mu} b_{L} H^{\dagger} i \overleftrightarrow{D}_{\mu} H$ & $\Lambda_{\mathrm{IR}} \gtrsim \mathbf{5} \sqrt{\boldsymbol{g}_{*} x_{\boldsymbol{t}}}(*)$ & $\Lambda_{\mathrm{IR}} \gtrsim 0.6 \sqrt{g_{*} x_{b}}$ & $\Lambda_{\mathrm{IR}} \gtrsim 0.6 \sqrt{g_{*} x_{b}}$ & $\Lambda_{\mathrm{IR}} \gtrsim 5 \sqrt{g_{*} x_{t}}$ \\
\hline Neutron EDM & $t$ partly-comp. & $b$ partly-comp. & $s$ partly-comp. & Anarchic \\
\hline $\bar{d}_{L} \sigma^{\mu \nu} e F_{\mu \nu} d_{R}$ & - & $\Lambda_{\mathrm{IR}} \gtrsim 0.24 g_{*} \sqrt{\alpha_{L}^{d b}}$ & $\Lambda_{\mathrm{IR}} \gtrsim 1.2 g_{*} \sqrt{\alpha_{L}^{d s}}$ & $\Lambda_{\mathrm{IR}} \gtrsim \mathbf{2 . 5} g_{*}$ \\
\hline $\bar{u}_{L} \sigma^{\mu \nu} e F_{\mu \nu} u_{R}$ & & & & $\Lambda_{\mathrm{IR}} \gtrsim 0.9 g_{*}$ \\
\hline $\bar{d}_{L} \sigma^{\mu \nu} g_{s} G_{\mu \nu} d_{R}$ & - & $\Lambda_{\mathrm{IR}} \gtrsim 0.3 g_{*} \sqrt{\alpha_{L}^{d b}}$ & $\Lambda_{\mathrm{IR}} \gtrsim 1.5 g_{*} \sqrt{\alpha_{L}^{d s}}$ & $\Lambda_{\mathrm{IR}} \gtrsim 3.2 g_{*}$ \\
\hline $\bar{u}_{L} \sigma^{\mu \nu} g_{s} G_{\mu \nu} u_{R}$ & . & & . & $\Lambda_{\mathrm{IR}} \gtrsim 1.2 g_{*}$ \\
\hline $\bar{c}_{L} \sigma^{\mu \nu} g_{s} G_{\mu \nu} c_{R}$ & . & & . & $\Lambda_{\mathrm{IR}} \gtrsim 1 g_{*}$ \\
\hline $\bar{b}_{L} \sigma^{\mu \nu} g_{s} G_{\mu \nu} b_{R}$ & - & $\Lambda_{\mathrm{IR}} \gtrsim 0.6 g_{*}$ & . & $\Lambda_{\mathrm{IR}} \gtrsim 0.6 g_{*}$ \\
\hline $\bar{t}_{L} \sigma^{\mu \nu} g_{s} G_{\mu \nu} t_{R}$ & $\Lambda_{\mathrm{IR}} \gtrsim 0.24 g_{*}$ & & . & $\Lambda_{\mathrm{IR}} \gtrsim 0.24 g_{*}$ \\
\hline Leptons & $t$ party comp. & $\tau$ partly-comp. & $\mu$ partly-comp. & Anarchic \\
\hline $\bar{e}_{L} \sigma^{\mu \nu} e F_{\mu \nu} e_{R}$ & $\Lambda_{\mathrm{IR}} \gtrsim 1.6 \sqrt{g_{*} x_{t}}$ & $\Lambda_{\mathrm{IR}} \gtrsim 0.5 g_{*} \sqrt{\alpha_{L}^{e \tau} \alpha_{R}^{e \tau}}$ & $\Lambda_{\mathrm{IR}} \gtrsim 2 g_{*} \sqrt{\alpha_{L}^{e \mu} \alpha_{R}^{e \mu}}$ & $\Lambda_{\mathrm{IR}} \gtrsim 32 g_{*}$ \\
\hline $\bar{\mu} \sigma^{\mu \nu} e F_{\mu \nu} e_{L, R}$ & . & $\Lambda_{\mathrm{IR}} \gtrsim 1.2 g_{*} \sqrt{\alpha_{L, R}^{e \tau} \alpha_{R, L}^{\mu \tau}}$ & $\Lambda_{\mathrm{IR}} \gtrsim 5 g_{*} \sqrt{\alpha_{L, R}^{e \mu}}$ & $\Lambda_{\mathrm{IR}} \gtrsim 19 g_{*}$ \\
\hline $\bar{\tau} \sigma^{\mu \nu} e F_{\mu \nu} \mu_{L, R}$ & . & $\Lambda_{\mathrm{IR}} \gtrsim 0.7 g_{*} \sqrt{\alpha_{L, R}^{\mu \tau}}$ & $\Lambda_{\mathrm{IR}} \gtrsim 1.3 g_{*}$ & $\Lambda_{\mathrm{IR}} \gtrsim 1.3 g_{*}$ \\
\hline $\bar{\tau} \sigma^{\mu \nu} e F_{\mu \nu} e_{L, R}$ & . & & $\Lambda_{\mathrm{IR}} \gtrsim 0.1 g_{*} \sqrt{\alpha_{L, R}^{e \mu}}$ & $\Lambda_{\mathrm{IR}} \gtrsim 0.4 g_{*}$ \\
\hline
\end{tabular}

Table 3. Bounds on $\Lambda_{\text {IR }}$ from assuming that the top, bottom, etc. are partly composite at $\Lambda_{\text {IR }}$. The results are given in TeV. Entries with a "." correspond to negligible bounds, while "_." means that the corresponding operator is not generated. The most relevant constraints are highlighted in boldface. If a custodial $P_{L R}$ symmetry [54] is present in the top mixings, the bounds denoted by $(*)$ are absent.

motivated by the lightness of the Higgs, are able to provide quantitative predictions depending only on how the global group $\mathcal{G}$ of the strong sector is broken. We will consider in particular the MCHM based on the $\mathcal{G} / \mathcal{H}=\mathrm{SO}(5) / \mathrm{SO}(4)$ coset $[57,58]$. Either in the case of partly-composite fermions at $\Lambda_{\mathrm{IR}}$ or at a larger scale $\Lambda_{f}$, the Higgs couplings depend on how the symmetry $\mathcal{G}$ is broken by eq. (1.1), and this is determined by specifying how $\mathcal{O}_{f_{i}}$ is embedded into a representation of $\mathcal{G}$. Therefore for both cases, the Higgs couplings to fermions can be written as

$$
\frac{g_{f f}^{h}}{g_{f f}^{h \mathrm{SM}}}=\frac{1-(1+n) v^{2} / f_{h}^{2}}{\sqrt{1-v^{2} / f_{h}^{2}}}
$$


where $n=0,1,2, \ldots$ and $f_{h}$ is the Higgs decay constant, $f_{h} \sim \Lambda_{\mathrm{IR}} / g_{*}$. For $\mathcal{O}_{f_{i}} \in \mathbf{4}$ (or $\mathbf{5}$ ) of $\mathrm{SO}(5)$, as in the MCHM4 (MCHM5), one finds $n=0(n=1)[43,59]$. This is also the case even if fermion masses come from bilinears $\bar{f}_{L} \mathcal{O}_{H} f_{R}$ with unknown UV origin. Indeed, in this case we need to specify into which representation of $\mathcal{G}$ we embed $\mathcal{O}_{H}$, or, equivalently, to specify an embedding for $\bar{f}_{L} f_{R}$. This latter can be formally written as a product of the representations of the individual embeddings for $\bar{f}_{L}$ and $f_{R}$. Therefore, by specifying these individual embeddings, we can determine again the Higgs couplings. As an example, let us consider $\mathcal{O}_{H} \in \mathbf{5}, \mathbf{1 4}$. Since $\mathbf{5} \in \overline{\mathbf{4}} \times \mathbf{4}$ and $\mathbf{1 4} \in \mathbf{5} \times \mathbf{5}$, we find respectively $n=0,1$, as in the MCHM4 and MCHM5.

It is also interesting to analyze the predictions for flavor-changing Higgs couplings. The coupling $h \bar{\tau} \mu$ is of special interest, as this is the one which experimental constraints have been presented from $h \rightarrow \tau \mu[60,61]$. We find however that contributions to this coupling are very small. For example, even for the case of a $\tau$ partly-composite at $\Lambda_{\mathrm{IR}}$, we get

$$
B R(h \rightarrow \mu \tau) \simeq\left(\frac{g_{*}^{2} v^{2}}{\Lambda_{\mathrm{IR}}^{2}} \frac{m_{\mu}}{m_{\tau}}\right)^{2} B R(h \rightarrow \tau \tau) \sim 2 \times 10^{-4}\left(\frac{g_{*} v}{\Lambda_{\mathrm{IR}}}\right)^{4},
$$

that is much below the present limit $B R(h \rightarrow \mu \tau)<1.51 \%$ from CMS [60] (1.85\% from ATLAS [61]). A larger effect is found if $\mu$ is partly composite at $\Lambda_{\mathrm{IR}}$ :

$$
B R(h \rightarrow \mu \tau) \simeq\left(\frac{g_{*}^{2} v^{2}}{\Lambda_{\mathrm{IR}}^{2}} \sqrt{\frac{m_{\mu}}{m_{\tau}}}\right)^{2} B R(h \rightarrow \tau \tau) \sim 4 \times 10^{-3}\left(\frac{g_{*} v}{\Lambda_{\mathrm{IR}}}\right)^{4} .
$$

This result is very close to the experimental bound, but we must in this case face the large contribution to $\mu \rightarrow e \gamma$ discussed above.

\section{$6 \quad$ Neutrino masses}

In this section we would like to comment on the possible origin of the neutrino masses in these scenarios. In principle, the origin of neutrino masses could be the same as the one discussed above for the other fermions, if right-handed neutrinos are introduced in the SM. Nevertheless, a simpler option is to assume that lepton number is broken at some UV scale $\Lambda_{\nu}$ by higher-dimensional operators:

$$
\frac{1}{\Lambda_{\nu}^{2 d_{H}-1}} \bar{L}^{c} \mathcal{O}_{H} \mathcal{O}_{H} L
$$

where $L$ generically denotes a left-handed lepton. Eq. (6.1) leads to neutrino masses of order

$$
m_{\nu} \simeq \frac{g_{*}^{2} v^{2}}{\Lambda_{\mathrm{IR}}}\left(\frac{\Lambda_{\mathrm{IR}}}{\Lambda_{\nu}}\right)^{2 d_{H}-1}
$$

For $d_{H}=2, g_{*} \sim 4 \pi$ and $\Lambda_{\mathrm{IR}} \sim 3 \mathrm{TeV}$, eq. (6.2) gives

$$
m_{\nu} \sim 0.1-0.01 \mathrm{eV} \quad \text { for } \quad \Lambda_{\nu} \sim 0.8-1.5 \times 10^{8} \mathrm{GeV} .
$$

This scale $\Lambda_{\nu}$ could be related to the scale at which other fermion masses are generated, for example, to $\Lambda_{s}$ or $\Lambda_{d}$. On the other hand, large mixing angles in the neutrino sector between two families can be easy obtained by requiring the corresponding neutrino masses to be generated at the same scale $\Lambda_{\nu}$. 


\section{Conclusions}

In this work we have proposed a new realization of the flavor structure in composite Higgs scenarios. The new construction is based on a departure from the usual partial compositeness framework for the light (i.e. not the top quark) SM fermions, both in the quark and lepton sector. The main idea is to assume that the light SM fermions get their mass through effective interactions involving fermion bilinears, namely operators of the form $\bar{f}_{L} \mathcal{O}_{H} f_{R}$, where $\mathcal{O}_{H}$ is a composite operator associated with the Higgs field. These Yukawa-like operators for the various fermion species are generated at hierarchically different energy scales $\Lambda_{f}$, thus effectively giving rise to the hierarchy of SM fermion masses and to the structure of the CKM matrix.

The only field that does not follow this construction is the top quark, whose large Yukawa coupling points towards a partial-compositeness origin at $\Lambda_{\mathrm{IR}} \sim \mathrm{TeV}$, the scale at which the Higgs emerges as a composite state. The left-handed and right-handed top components are thus linearly mixed with suitable composite operators, $\epsilon_{f_{i}} \bar{f}_{i} \mathcal{O}_{f_{i}}$, following the usual anarchic flavor structure.

The new framework leads to a significant improvement of the compatibility of the composite Higgs models with the flavor constraints. The most remarkable difference with respect to the anarchic scenarios is the suppression of new-physics effects in dipole operators. The most severe bounds of the anarchic scenario, namely the ones coming from the neutron and electron EDMs and from $\mu \rightarrow e \gamma$, are absent in the new framework (see table 3$)$.

The most important contributions in our scenario come from two flavor-violating operators arising from the top partial compositeness. Up to an unknown coefficient expected to be of order one, these are given by

where

$$
\frac{1}{\Lambda_{\mathrm{IR}}^{2}}\left(g_{i j} \bar{d}_{L i} \gamma^{\mu} d_{L j}\right)^{2}, \quad \frac{g_{*} v^{2}}{\Lambda_{\mathrm{IR}}^{2}} g_{i j}\left(\bar{d}_{L i} \gamma^{\mu} d_{L j}\right) \frac{g Z_{\mu}}{\cos \theta_{W}}
$$

$$
g_{i j} \equiv Y_{t} x_{t}\left(V_{\mathrm{CKM}}^{\dagger}\right)_{i 3}\left(V_{\mathrm{CKM}}\right)_{3 j},
$$

and $d_{L i}$ denotes the left-handed down-type quark component in the $i$-th family. A remarkable feature of these corrections is the fact that they automatically follow a MFV structure. The first operator contributes to $\Delta F=2$ transitions and generates correlated effects in the $\epsilon_{K}, \Delta M_{B_{d}}$ and $\Delta M_{B_{s}}$ observables, which are of the order of the present experimental sensitivity if we take $\Lambda_{\mathrm{IR}} \sim \mathrm{TeV}$ and we allow for a slight reduction of the left-handed top compositeness, $x_{t}<1$. The second operator of eq. (7.1) gives flavor-changing $Z$-couplings. At present it only pushes the $\Lambda_{\mathrm{IR}}$ scale in the few $\mathrm{TeV}$ range. In the future it can be seen either in deviations in the decays $K \rightarrow \mu \mu$ or $B \rightarrow(X) \ell \ell$. This contribution can however be significantly smaller if the strong sector is invariant under a custodial $P_{L R}$ symmetry, which protects the down-type quark couplings to the $Z$ boson [54].

Additional contributions to $\Delta F=2$ operators can also be generated at the scales $\Lambda_{c, s, d}$ at which the second and first family quarks get their masses. These corrections however only give a sizable effect on $\epsilon_{K}$ for $\Lambda_{\mathrm{IR}}$ below the multi-TeV range, a much smaller contribution than the anarchic one. It must however be stressed that these bounds depend on the 


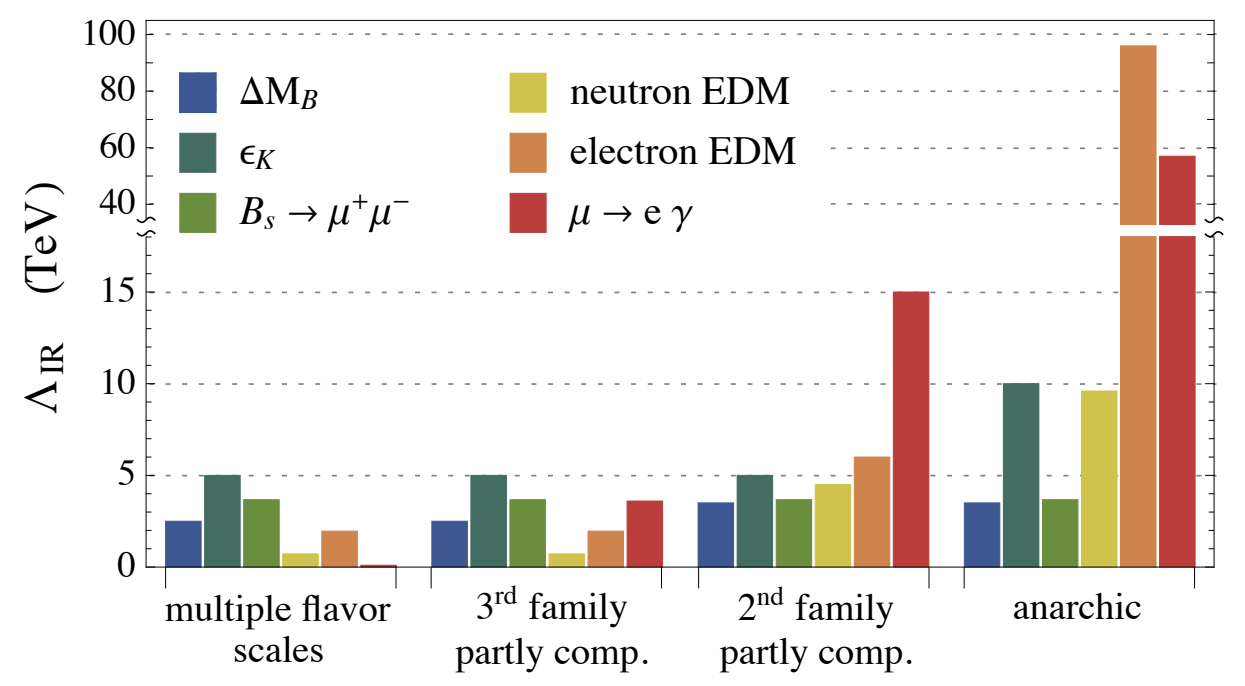

Figure 4. Lower bounds on $\Lambda_{\mathrm{IR}}$ on the various flavor scenarios. The first set of bounds corresponds to our scenario with multiple flavor scales, the second and third sets assume partial compositeness at $\Lambda_{\mathrm{IR}}$ for the whole third and second family respectively, while the last set gives the bounds for the anarchic flavor scenario. To derive the numerical values we have taken $g_{*} \simeq 3, x_{t} \simeq x_{c} \simeq 0.5$, and set all free $\alpha_{L, R}$ parameters to one.

coefficients of the effective operators which are affected by some degree of uncertainty. These contributions to $\epsilon_{K}$ severely constrain the maximal dimension of the $\mathcal{O}_{H}$ operator, requiring $d_{H} \lesssim 2$.

We also considered possible variations of the framework described above. For example, a more economical scenario has been proposed in which each family is associated to a single flavor scale at which the bilinear mass operators are generated. A few additional new-physics flavor effects are generated in this case, which are of the same order of the experimental bounds. In particular, assuming $\tau$ partial compositeness at $\Lambda_{\mathrm{IR}}$ (as the top and bottom) leads to corrections to the electron EDM and to the lepton-number violating processes $\mu \rightarrow e \gamma$ and $\tau \rightarrow \mu \gamma$ which could be visible in forthcoming experiments. On the other hand, reducing down to $\Lambda_{\mathrm{IR}}$ the scale at which the Yukawa interactions are generated for the second family seems disfavored, since it leads to large corrections to the neutron and electron EDMs as well as to $\mu \rightarrow e \gamma$.

Finally, we have also presented the size of deviations in Higgs couplings, eq. (5.1), predictions for $h \rightarrow \tau \mu$, and discussed the possible origin of the neutrino masses.

A comparison of the bounds in the various scenarios we considered in our analysis is shown in figure 4 for a typical choice of parameters. We have also included for comparison the constraints for the anarchic flavor scenario. Figure 4 shows the main point of the article: there are natural scenarios where the origin of flavor and electroweak scale can be determined dynamically, and where, without tuning or imposing extra symmetries, contributions to flavor and CP-violating observables can still be below (or better say, saturating) the present bounds, providing then a motivation for an experimental improvement in the near future. 


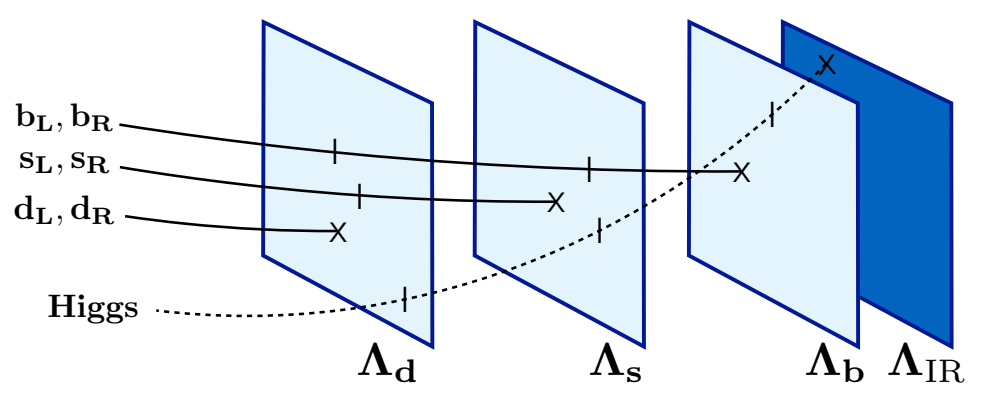

Figure 5. Five-dimensional model which, by AdS/CFT, corresponds to a model of flavor for the down sector and Higgs of the SM giving the same Yukawa structure as eq. (2.7).

\section{Acknowledgments}

We thank Jernej Kamenik and Andrea Wulzer for useful discussions, and especially Riccardo Rattazzi for a critical reading of our paper. This work has been partly supported by the Catalan ICREA Academia Program, and grants FPA2014-55613-P, 2014-SGR-1450 and Severo Ochoa excellence program SO-2012-0234.

\section{A Warped five-dimensional models with multiple flavor scales}

For AdS/CFT practitioners it can be useful to depict warped five-dimensional models which, by means of the AdS/CFT correspondence, lead to the scenarios of flavor considered above.

As an example, we consider a model for the down-type quark sector and Higgs of the SM. This is shown in figure 5. It corresponds to a warped extra dimension with 3 branes located at different positions and therefore associated with 3 different energy scales $\Lambda_{d, s, b}$. We assume that only one left-handed and right-handed quark can propagate up to the brane at $\Lambda_{b}$, what we call the bottom quark, while two can propagate up to the brane at $\Lambda_{s}$. On the other hand, the three quarks can be present on the brane at $\Lambda_{d}$. The warped extra dimension extends up to the brane at $\Lambda_{\mathrm{IR}}$. The Higgs arises from a $5 \mathrm{D}$ scalar field whose zero-mode is mostly localized at $\Lambda_{\mathrm{IR}}$, as shown with a dashed line in figure 5 (the more localized towards $\Lambda_{\mathrm{IR}}$, corresponds to larger values of $d_{H}$ ). Possible examples of wave-functions for the zero-modes of the quarks are also shown in figure 5 with solid lines. Yukawa couplings come from the overlapping of zero-mode wave-functions. The small overlapping of the Higgs wave-function with those of the quarks localized far away from $\Lambda_{\mathrm{IR}}$ would explain the smallness of these Yukawa couplings. The generalization to the up and lepton sector is straightforward. If the up sector is included, one has to assure that the left-handed doublets reach also the corresponding branes where the up-type quark masses are generated, e.g., $Q_{L 3}=\left(t_{L}, b_{L}\right)^{T}$ must reach $\Lambda_{t}$.

\section{B Mixing angles in the $\alpha_{L}^{d s, d b, s b} \approx 0$ limit}

Although the elements of $V_{L}^{\text {down }}$ are fixed by the requirement of reproducing the CKM structure, the elements of $V_{R}^{\text {down }}$ are free parameters and could be substantially reduced. 
In this appendix we want to show how small the off-diagonal entries of $V_{R}^{\text {down }}$ could be in the situation where $\alpha_{L} \sim 0$.

Having $\alpha_{L} \sim 0$ could arise from certain accidental symmetries at $\Lambda_{d, s}$. For example, if at $\Lambda_{d}$ there is a $Z_{2}$ symmetry under which $s_{L}$ and $b_{L}$ are odd, this would imply $\epsilon_{s_{L}, b_{L}}^{(1)}=0$. Similarly, if at $\Lambda_{s}$ this $Z_{2}$ parity is still preserved but only for $b_{L}$, we would have $\epsilon_{b_{L}}^{(2)}=0$. This would give $\alpha_{L}^{d s}=\alpha_{L}^{d b}=\alpha_{L}^{s b}=0$. This accidental $Z_{2}$ parity could arise from the dynamics of the model. For example, if $b_{L}$ is mostly composite at $\Lambda_{\mathrm{IR}}$, its couplings at $\Lambda_{d, s}$ will be suppressed (in warped five-dimensional models this implies that the wave-function of $b_{L}$ is peaked toward the $\Lambda_{\mathrm{IR}}$ brane, having a small overlapping with the $\Lambda_{d, s}$ branes).

Having $\alpha_{L} \sim 0$ leads to a $V_{R}^{\text {down }}$ different from eq. (2.13), where $\alpha_{L} \sim 1$ was assumed. For example, in the case $\alpha_{L}^{d s}=\alpha_{L}^{d b}=0$ and $\alpha_{L}^{s b} \sim 1$ we get

$$
\begin{aligned}
& \left(V_{R}^{\text {down }}\right)_{31} \sim \alpha_{R}^{d s} \alpha_{L}^{s b} \frac{Y_{d}^{2}}{Y_{s} Y_{b}} \simeq\left(V_{\mathrm{CKM}}\right)_{21} \alpha_{L}^{s b} \frac{Y_{d}}{Y_{b}} \simeq \lambda_{c} \alpha_{L}^{s b} \frac{Y_{d}}{Y_{b}} \\
& \left(V_{R}^{\text {down }}\right)_{13} \sim \alpha_{R}^{d b}\left(\frac{Y_{d}}{Y_{b}}\right)^{2} \simeq\left(V_{\mathrm{CKM}}\right)_{31} \frac{Y_{d}}{Y_{b}} \simeq \lambda_{c}^{3} \frac{Y_{d}}{Y_{b}} \\
& \left(V_{R}^{\text {down }}\right)_{12} \sim\left(V_{R}^{\text {down }}\right)_{21} \sim \alpha_{R}^{d s}\left(\frac{Y_{d}}{Y_{s}}\right)^{2} \simeq\left(V_{\mathrm{CKM}}\right)_{21} \frac{Y_{d}}{Y_{s}} \simeq \lambda_{c} \frac{Y_{d}}{Y_{s}} .
\end{aligned}
$$

Notice that in this case the entries involving the first and third families are not symmetric, that is $\left(V_{R}^{\text {down }}\right)_{13} \gg\left(V_{R}^{\text {down }}\right)_{31}$. If $\alpha_{L}^{s b}=0$ as well, the estimate for $\left(V_{R}^{\text {down }}\right)_{31}$ coincides with the one for $\left(V_{R}^{\text {down }}\right)_{13}$, namely

$$
\left(V_{R}^{\text {down }}\right)_{31} \sim\left(V_{R}^{\text {down }}\right)_{13} \sim \lambda_{c}^{3} \frac{Y_{d}}{Y_{b}} .
$$

Analogous results can be found for the $V_{L, R}^{\mathrm{up}}$ matrices.

Open Access. This article is distributed under the terms of the Creative Commons Attribution License (CC-BY 4.0), which permits any use, distribution and reproduction in any medium, provided the original author(s) and source are credited.

\section{References}

[1] R. Rattazzi, V.S. Rychkov, E. Tonni and A. Vichi, Bounding scalar operator dimensions in 4D CFT, JHEP 12 (2008) 031 [arXiv:0807.0004] [InSPIRE].

[2] D.B. Kaplan, Flavor at SSC energies: A New mechanism for dynamically generated fermion masses, Nucl. Phys. B 365 (1991) 259 [INSPIRE].

[3] Y. Grossman and M. Neubert, Neutrino masses and mixings in nonfactorizable geometry, Phys. Lett. B 474 (2000) 361 [hep-ph/9912408] [INSPIRE].

[4] T. Gherghetta and A. Pomarol, Bulk fields and supersymmetry in a slice of AdS, Nucl. Phys. B 586 (2000) 141 [hep-ph/0003129] [INSPIRE].

[5] S.J. Huber and Q. Shafi, Fermion masses, mixings and proton decay in a Randall-Sundrum model, Phys. Lett. B 498 (2001) 256 [hep-ph/0010195] [INSPIRE]. 
[6] S.J. Huber, Flavor violation and warped geometry, Nucl. Phys. B 666 (2003) 269 [hep-ph/0303183] [INSPIRE].

[7] K. Agashe, G. Perez and A. Soni, Flavor structure of warped extra dimension models, Phys. Rev. D 71 (2005) 016002 [hep-ph/0408134] [INSPIRE].

[8] B. Keren-Zur, P. Lodone, M. Nardecchia, D. Pappadopulo, R. Rattazzi and L. Vecchi, On Partial Compositeness and the CP asymmetry in charm decays, Nucl. Phys. B 867 (2013) 394 [arXiv: 1205.5803] [INSPIRE].

[9] C. Csáki, A. Falkowski and A. Weiler, The Flavor of the Composite Pseudo-Goldstone Higgs, JHEP 09 (2008) 008 [arXiv:0804.1954] [INSPIRE].

[10] C. Csáki, A. Falkowski and A. Weiler, A Simple Flavor Protection for RS, Phys. Rev. D 80 (2009) 016001 [arXiv:0806.3757] [InSPIRE].

[11] O. Gedalia, G. Isidori and G. Perez, Combining Direct \&3 Indirect Kaon CP-violation to Constrain the Warped KK Scale, Phys. Lett. B 682 (2009) 200 [arXiv:0905.3264] [INSPIRE].

[12] K. Agashe and R. Contino, Composite Higgs-Mediated FCNC, Phys. Rev. D 80 (2009) 075016 [arXiv: 0906.1542] [inSPIRE].

[13] N. Vignaroli, $\Delta F=1$ constraints on composite Higgs models with LR parity, Phys. Rev. D 86 (2012) 115011 [arXiv:1204.0478] [INSPIRE].

[14] R. Barbieri, D. Buttazzo, F. Sala, D.M. Straub and A. Tesi, A 125 GeV composite Higgs boson versus flavour and electroweak precision tests, JHEP 05 (2013) 069 [arXiv: 1211.5085] [INSPIRE].

[15] M. Bauer, S. Casagrande, U. Haisch and M. Neubert, Flavor Physics in the Randall-Sundrum Model: II. Tree-Level Weak-Interaction Processes, JHEP 09 (2010) 017 [arXiv: 0912.1625] [INSPIRE].

[16] M. König, M. Neubert and D.M. Straub, Dipole operator constraints on composite Higgs models, Eur. Phys. J. C 74 (2014) 2945 [arXiv:1403.2756] [INSPIRE].

[17] G. Panico and A. Wulzer, The Composite Nambu-Goldstone Higgs, Lect. Notes Phys. 913 (2016) 1 [arXiv: 1506.01961] [INSPIRE].

[18] C. Csáki, M. Geller, O. Telem and A. Weiler, The Flavor of the Composite Twin Higgs, arXiv: 1512.03427 [INSPIRE].

[19] D. Croon, B.M. Dillon, S.J. Huber and V. Sanz, Exploring holographic Composite Higgs models, arXiv:1510.08482 [INSPIRE].

[20] M. Redi and A. Weiler, Flavor and CP Invariant Composite Higgs Models, JHEP 11 (2011) 108 [arXiv: 1106.6357] [INSPIRE].

[21] M. Redi, Composite MFV and Beyond, Eur. Phys. J. C 72 (2012) 2030 [arXiv:1203.4220] [INSPIRE].

[22] G. Isidori, Flavour Physics and Implication for New Phenomena, arXiv:1507.00867 [INSPIRE].

[23] O. Domenech, A. Pomarol and J. Serra, Probing the SM with Dijets at the LHC, Phys. Rev. D 85 (2012) 074030 [arXiv:1201.6510] [INSPIRE].

[24] M. Redi, V. Sanz, M. de Vries and A. Weiler, Strong Signatures of Right-Handed Compositeness, JHEP 08 (2013) 008 [arXiv: 1305.3818] [INSPIRE]. 
[25] C. Delaunay, T. Flacke, J. Gonzalez-Fraile, S.J. Lee, G. Panico and G. Perez, Light Non-degenerate Composite Partners at the LHC, JHEP 02 (2014) 055 [arXiv:1311.2072] [INSPIRE].

[26] R. Rattazzi and A. Zaffaroni, Comments on the holographic picture of the Randall-Sundrum model, JHEP 04 (2001) 021 [hep-th/0012248] [INSPIRE].

[27] G. Cacciapaglia, C. Csáki, J. Galloway, G. Marandella, J. Terning and A. Weiler, A GIM Mechanism from Extra Dimensions, JHEP 04 (2008) 006 [arXiv:0709.1714] [INSPIRE].

[28] J. Santiago, Minimal Flavor Protection: A New Flavor Paradigm in Warped Models, JHEP 12 (2008) 046 [arXiv:0806.1230] [INSPIRE].

[29] R. Barbieri, G. Isidori and D. Pappadopulo, Composite fermions in Electroweak Symmetry Breaking, JHEP 02 (2009) 029 [arXiv:0811.2888] [INSPIRE].

[30] C. Delaunay, O. Gedalia, S.J. Lee, G. Perez and E. Ponton, Ultra Visible Warped Model from Flavor Triviality and Improved Naturalness, Phys. Rev. D 83 (2011) 115003 [arXiv: 1007.0243] [INSPIRE].

[31] C. Delaunay, O. Gedalia, S.J. Lee, G. Perez and E. Ponton, Extraordinary Phenomenology from Warped Flavor Triviality, Phys. Lett. B 703 (2011) 486 [arXiv:1101.2902] [INSPIRE].

[32] R. Barbieri, D. Buttazzo, F. Sala and D.M. Straub, Flavour physics from an approximate $\mathrm{U}(2)^{3}$ symmetry, JHEP 07 (2012) 181 [arXiv: 1203.4218] [INSPIRE].

[33] S.M. Barr and A. Zee, Electric Dipole Moment of the Electron and of the Neutron, Phys. Rev. Lett. 65 (1990) 21 [Erratum ibid. 65 (1990) 2920] [INSPIRE].

[34] S. Dimopoulos and L. Susskind, Mass Without Scalars, Nucl. Phys. B 155 (1979) 237 [INSPIRE].

[35] E. Eichten and K.D. Lane, Dynamical Breaking of Weak Interaction Symmetries, Phys. Lett. B 90 (1980) 125 [INSPIRE].

[36] Z.G. Berezhiani and R. Rattazzi, Inverse hierarchy approach to fermion masses, Nucl. Phys. B 407 (1993) 249 [hep-ph/9212245] [INSPIRE].

[37] O. Matsedonskyi, On Flavour and Naturalness of Composite Higgs Models, JHEP 02 (2015) 154 [arXiv:1411.4638] [INSPIRE].

[38] G. Cacciapaglia, H. Cai, T. Flacke, S.J. Lee, A. Parolini and H. Serôdio, Anarchic Yukawas and top partial compositeness: the flavour of a successful marriage, JHEP 06 (2015) 085 [arXiv: 1501.03818] [INSPIRE].

[39] O. Matsedonskyi, G. Panico and A. Wulzer, Light Top Partners for a Light Composite Higgs, JHEP 01 (2013) 164 [arXiv: 1204.6333] [INSPIRE].

[40] M. Redi and A. Tesi, Implications of a Light Higgs in Composite Models, JHEP 10 (2012) 166 [arXiv: 1205.0232] [inSPIRE].

[41] D. Marzocca, M. Serone and J. Shu, General Composite Higgs Models, JHEP 08 (2012) 013 [arXiv: 1205.0770] [INSPIRE].

[42] G. Panico, M. Redi, A. Tesi and A. Wulzer, On the Tuning and the Mass of the Composite Higgs, JHEP 03 (2013) 051 [arXiv:1210.7114] [INSPIRE].

[43] A. Pomarol and F. Riva, The Composite Higgs and Light Resonance Connection, JHEP 08 (2012) 135 [arXiv:1205.6434] [INSPIRE]. 
[44] UTfit collaboration, M. Bona et al., Model-independent constraints on $\Delta F=2$ operators and the scale of new physics, JHEP 03 (2008) 049 [arXiv:0707.0636] [INSPIRE].

[45] W. Altmannshofer and D.M. Straub, Cornering New Physics in $b \rightarrow s$ Transitions, JHEP 08 (2012) 121 [arXiv:1206.0273] [INSPIRE].

[46] D. Guadagnoli and G. Isidori, $B\left(B_{s} \rightarrow \mu^{+} \mu^{-}\right)$as an electroweak precision test, Phys. Lett. B 724 (2013) 63 [arXiv: 1302.3909] [INSPIRE].

[47] A.J. Buras, C. Grojean, S. Pokorski and R. Ziegler, FCNC Effects in a Minimal Theory of Fermion Masses, JHEP 08 (2011) 028 [arXiv: 1105.3725] [INSPIRE].

[48] C. Grojean, O. Matsedonskyi and G. Panico, Light top partners and precision physics, JHEP 10 (2013) 160 [arXiv: 1306.4655] [INSPIRE].

[49] D. Ghosh, M. Salvarezza and F. Senia, Extending the Analysis of Electroweak Precision Constraints in Composite Higgs Models, arXiv:1511.08235 [INSPIRE].

[50] Particle Data Group collaboration, K.A. Olive et al., Review of Particle Physics, Chin. Phys. C 38 (2014) 090001 [INSPIRE].

[51] D. Chang, W.-Y. Keung, C.S. Li and T.C. Yuan, QCD Corrections to CP Violation From Color Electric Dipole Moment of b Quark, Phys. Lett. B 241 (1990) 589 [INSPIRE].

[52] J.F. Kamenik, M. Papucci and A. Weiler, Constraining the dipole moments of the top quark, Phys. Rev. D 85 (2012) 071501(R) [Erratum ibid. D 88 (2013) 039903] [arXiv:1107.3143] [INSPIRE].

[53] F. Sala, A bound on the charm chromo-EDM and its implications, JHEP 03 (2014) 061 [arXiv: 1312.2589] [INSPIRE].

[54] K. Agashe, R. Contino, L. Da Rold and A. Pomarol, A Custodial symmetry for Z $b \bar{b}$, Phys. Lett. B 641 (2006) 62 [hep-ph/0605341] [INSPIRE].

[55] J. Brod, U. Haisch and J. Zupan, Constraints on CP-violating Higgs couplings to the third generation, JHEP 11 (2013) 180 [arXiv:1310.1385] [INSPIRE].

[56] M. Beneke, P. Moch and J. Rohrwild, Lepton flavour violation in RS models with a brane- or nearly brane-localized Higgs, Nucl. Phys. B 906 (2016) 561 [arXiv:1508.01705] [InSPIRE].

[57] K. Agashe, R. Contino and A. Pomarol, The Minimal composite Higgs model, Nucl. Phys. B 719 (2005) 165 [hep-ph/0412089] [INSPIRE].

[58] R. Contino, L. Da Rold and A. Pomarol, Light custodians in natural composite Higgs models, Phys. Rev. D 75 (2007) 055014 [hep-ph/0612048] [INSPIRE].

[59] G.F. Giudice, C. Grojean, A. Pomarol and R. Rattazzi, The Strongly-Interacting Light Higgs, JHEP 06 (2007) 045 [hep-ph/0703164] [INSPIRE].

[60] CMS collaboration, Search for Lepton-Flavour-Violating Decays of the Higgs Boson, Phys. Lett. B 749 (2015) 337 [arXiv: 1502.07400] [INSPIRE].

[61] ATLAS collaboration, Search for lepton-flavour-violating $H \rightarrow \mu \tau$ decays of the Higgs boson with the ATLAS detector, JHEP 11 (2015) 211 [arXiv:1508.03372] [INSPIRE]. 